\title{
Calculation of improper integrals using uniformly distributed sequences
}

by

\author{
Christoph Baxa (Wien)
}

Dedicated to Professor Wolfgang M. Schmidt on the occasion of his seventieth birthday

\section{Introduction}

1.1. History of the problem. It is a basic result of the theory of uniformly distributed sequences that a sequence $\left(\omega_{k}\right)_{k \geq 1}$ is uniformly distributed in $[0,1]$ if and only if

$$
\lim _{N \rightarrow \infty} \frac{1}{N} \sum_{k=1}^{N} f\left(\omega_{k}\right)=\int_{0}^{1} f(x) d x
$$

for all Riemann-integrable functions $f:[0,1] \rightarrow \mathbb{R}$. However, the analogous equivalence is not true for Lebesgue-integrable functions. (This can be seen by setting $f=c_{\Omega}$ where $\Omega=\left\{\omega_{k} \mid k \in \mathbb{N}\right\}$ and $c_{M}$ denotes the characteristic function of a set $M \subseteq \mathbb{R}$.) It was even pointed out by N. G. de Bruijn and K. A. Post [2] that if (1.1) holds for all uniformly distributed sequences $\left(\omega_{k}\right)_{k \geq 1}$ then $f$ has to be Riemann-integrable.

On the other hand, if $\alpha$ is irrational and $f$ is an $L^{1}$-function then

$$
\lim _{N \rightarrow \infty} \frac{1}{N} \sum_{k=1}^{N} f(\{k \alpha+\xi\})=\int_{0}^{1} f(x) d x
$$

for almost all $\xi$ (where $\{x\}$ denotes the fractional part of $x \in \mathbb{R}$ ). This follows from Birkhoff's ergodic theorem but was also proved by A. Khinchin [9]. This example shows that it is an interesting problem to find conditions for relation (1.1) to hold if $\int_{0}^{1} f(x) d x$ exists only as an improper Riemann integral. (Results of this kind can be applied to the calculation of such integrals; see, for example, [19], [10] and [7].) For a more complete survey of

2000 Mathematics Subject Classification: Primary 11K31, 11J71, 26A42. 
the existing literature we refer the reader to the monographs by L. Kuipers and H. Niederreiter [12] and M. Drmota and R. F. Tichy [4].

In this paper we study the special case $\left(\omega_{k}\right)_{k \geq 1}=(\{k \alpha\})_{k \geq 1}$ with irrational $\alpha$. We find relations between certain non-Riemann-integrable functions $f:[0,1] \rightarrow \mathbb{R}$ and irrational $\alpha$ that are necessary or sufficient conditions for the relation

$$
\lim _{N \rightarrow \infty} \frac{1}{N} \sum_{k=1}^{N} f(\{k \alpha\})=\int_{0}^{1} f(x) d x
$$

to be true. The first results in this direction were obtained by G. H. Hardy and J. E. Littlewood [6]. Supposing that $\lim _{x \rightarrow 0+} f(x)=\lim _{x \rightarrow 1-} f(x)=$ $+\infty$ and that $f$ is Riemann-integrable on $[\delta, 1-\delta]$ for all $\delta \in(0,1 / 2)$, they proved that (1.2) holds for almost all $\alpha$ if

$$
\int_{0}^{1} f(x)\left(\log ^{2} \frac{1}{x}+\log ^{2} \frac{1}{1-x}\right) d x<\infty
$$

and that (1.2) holds if the continued fraction expansion of $\alpha$ is bounded. Some years earlier A. Khinchin [8] had posed the following question: if $E \subseteq$ $(0,1)$ has Lebesgue measure $\lambda(E)$ is it then true that

$$
\lim _{N \rightarrow \infty} \frac{1}{N} \sum_{k=1}^{N} c_{E}(\{k \alpha\})=\lambda(E)
$$

for almost all $\alpha$ ? This conjecture was disproved by J. M. Marstrand [14]. K. A. Driver, D. S. Lubinsky, G. Petruska and P. Sarnak [3] constructed functions for which (1.2) does not hold to study the radius of convergence of hypergeometric functions. V. A. Oskolkov [15] proved that (1.2) holds if and only if

$$
\lim _{m \rightarrow \infty} \frac{1}{q_{m}} f\left(\left\{q_{m} \alpha\right\}\right)=0
$$

where $f$ satisfies the same conditions as in [6] and $q_{m}$ denotes the denominator of the $m$ th convergent of the continued fraction expansion of $\alpha$. (In a follow-up paper [16] he proved a similar result for sequences satisfying a certain technical condition. In the case of sequences of shape $(\{k \alpha\})_{k \geq 1}$ this condition is equivalent to the boundedness of the continued fraction expansion of $\alpha$.) In a joint paper with J. Schoißengeier [1] we proved the following generalization of Oskolkov's result:

Theorem 1.1. Let $\alpha$ be an irrational number, $F \subseteq[0,1] \cap \mathbb{Q}$ finite, $f:[0,1] \rightarrow \mathbb{R}$ integrable, continuous almost everywhere and locally bounded in $[0,1] \backslash F$. Assume further that for every $\beta \in F$ there is some neighbourhood $U$ of $\beta$ such that $f$ is either bounded or monotone in $[0, \beta) \cap U$ and in $(\beta, 1] \cap U$ as well. Then the following are equivalent: 
(1) $\lim _{N \rightarrow \infty} f(\{N \alpha\}) / N=0$,

(2) $\lim _{N \rightarrow \infty} N^{-1} \sum_{k=1}^{N} f(\{k \alpha\})$ exists,

(3) $\lim _{N \rightarrow \infty} N^{-1} \sum_{k=1}^{N} f(\{k \alpha\})=\int_{0}^{1} f(x) d x$.

The implication $(3) \Rightarrow(2)$ is trivial and the implication $(2) \Rightarrow(1)$ is Lemma 1.2 below. However, it is much harder to prove $(1) \Rightarrow(3)$.

Lemma 1.2. Let $\alpha$ be irrational and $f:[0,1] \rightarrow \mathbb{R}$ any function. If $\lim _{N \rightarrow \infty} N^{-1} \sum_{k=1}^{N} f(\{k \alpha\})$ exists then $\lim _{N \rightarrow \infty} f(\{N \alpha\}) / N=0$.

Proof. This follows from

$$
\frac{1}{N} f(\{N \alpha\})=\frac{1}{N} \sum_{k=1}^{N} f(\{k \alpha\})-\frac{N-1}{N} \frac{1}{N-1} \sum_{k=1}^{N-1} f(\{k \alpha\}) \rightarrow 0
$$

as $N \rightarrow \infty$.

In Theorem 1.1 we assumed that the singularities of the function $f$ are all at rational points $\beta$ and we made crucial use of this assumption in the proof of the implication $(1) \Rightarrow(3)$. It is the purpose of the present paper to describe generalizations of Theorem 1.1 without this assumption. Comparison with the paper's precursor [1] will show the reader that we have reused many ideas and Theorem 1.1 is contained in our results as a special case. However, our generalizations are far from straightforward and finding them required a careful analysis and further developments of the proofs given in [1].

1.2. Basic assumptions and plan of the paper. For the remainder of the paper we assume that $\alpha$ is an irrational number with regular continued fraction expansion $\alpha=\left[a_{0}, a_{1}, a_{2}, \ldots\right]$ with convergents $p_{m} / q_{m}=\left[a_{0}, a_{1}, \ldots, a_{m}\right]$ for $m \geq 0$. The quantities $a_{m}, p_{m}$ and $q_{m}$ depend on $\alpha$ but we will usually suppress this fact in our notation. However, in Section 4 we will sometimes write $a_{m}(\alpha), p_{m}(\alpha)$ and $q_{m}(\alpha)$ when there is a danger of confusion.

We will now describe the class of functions we are going to deal with. Let $\beta \in(0,1]$. We will say the function $f:[0,1] \rightarrow \mathbb{R}$ is in the set $\mathcal{S}_{-}^{+}(\beta)$ if it satisfies the following conditions: $f \geq 0,\left.f\right|_{[0, \beta)}$ is increasing, $\lim _{x \rightarrow \beta-} f(x)$ $=+\infty,\left.f\right|_{[\beta, 1]}=0$ and $\int_{0}^{1} f(x) d x$ exists.

We define $f \in \mathcal{S}_{-}^{-}(\beta): \Leftrightarrow-f \in \mathcal{S}_{-}^{+}(\beta)$. For any function $f:[0,1] \rightarrow \mathbb{R}$ we set $\widehat{f}:[0,1] \rightarrow \mathbb{R}, \widehat{f}(x)=f(1-x)$. Obviously $\widehat{f}(\{k(-\alpha)\})=f(\{k \alpha\})$ for all positive integers $k$ and if $\int_{0}^{1} f(x) d x$ exists then so does $\int_{0}^{1} \widehat{f}(x) d x$ and its value is the same. For $\beta \in[0,1)$ we define $f \in \mathcal{S}_{+}^{+}(\beta): \Leftrightarrow \widehat{f} \in \mathcal{S}_{-}^{+}(1-\beta)$ and $f \in \mathcal{S}_{+}^{-}(\beta): \Leftrightarrow \widehat{f} \in \mathcal{S}_{-}^{-}(1-\beta)$. Finally, we call a function $f:[0,1] \rightarrow \mathbb{R}$ admissible if there exist finite (potentially empty) sets $\left\{\beta_{1}^{-}, \ldots, \beta_{r}^{-}\right\} \subseteq(0,1]$ and $\left\{\beta_{1}^{+}, \ldots, \beta_{s}^{+}\right\} \subseteq[0,1)$, a Riemann-integrable function $f_{0}:[0,1] \rightarrow \mathbb{R}$, functions $f_{i}^{-} \in \mathcal{S}_{-}^{+}\left(\beta_{i}^{-}\right) \cup \mathcal{S}_{-}^{-}\left(\beta_{i}^{-}\right)$for $1 \leq i \leq r$ and functions $f_{j}^{+} \in \mathcal{S}_{+}^{+}\left(\beta_{j}^{+}\right) \cup$ 
$\mathcal{S}_{+}^{-}\left(\beta_{j}^{+}\right)$for $1 \leq j \leq s$ such that

$$
f=f_{0}+f_{1}^{-}+\cdots+f_{r}^{-}+f_{1}^{+}+\cdots+f_{s}^{+} .
$$

For the sake of clarity we stress that $\beta_{1}^{-}, \ldots, \beta_{r}^{-}$are assumed to be $r$ pairwise different points and likewise $\beta_{1}^{+}, \ldots, \beta_{s}^{+}$are $s$ pairwise different points. However, it is possible that $\beta_{i}^{-}=\beta_{j}^{+}$for some $i$ and $j$. Our definition of an admissible function may seem clumsy when compared to the assumptions made about $f$ in Theorem 1.1. Yet, it is more general, which can be seen from the following example: let $f=f_{0}+f_{1}^{-}$where

$$
f_{0}(x)= \begin{cases}-1 / n & \text { if } x=(n-1) / n \text { for some positive integer } n \\ 0 & \text { otherwise }\end{cases}
$$

and

$$
f_{1}^{-}(x)= \begin{cases}1 / \sqrt{1-x} & \text { if } x \in[0,1), \\ 0 & \text { if } x=1 .\end{cases}
$$

In fact, Theorem 1.1 remains true if $f$ is assumed to be admissible.

The plan of this paper is the following: in Section 2 we collect auxiliary results about the distribution of the sequence $(k \alpha)_{k \geq 1}$ modulo 1 . Section 3 is the core of the paper. In it we establish a number of results about functions $f \in \mathcal{S}_{-}^{+}(\beta)$. The main results are:

- If $\lim _{N \rightarrow \infty} f(\{N \alpha\}) / N=0$ then

$$
\varliminf_{N \rightarrow \infty} \frac{1}{N} \sum_{k=1}^{N} f(\{k \alpha\})=\lim _{m \rightarrow \infty} \frac{1}{q_{m}} \sum_{k=1}^{q_{m}} f(\{k \alpha\})=\int_{0}^{1} f(x) d x .
$$

- Theorem 1.1 remains true if any of the following (rather strong) assumptions hold: the continued fraction expansion of $\alpha$ is bounded, $\beta$ is rational or $\varliminf_{m \rightarrow \infty}\left\|\beta q_{m}\right\|>0$.

- Suppose that $\lim _{N \rightarrow \infty} f(\{N \alpha\}) / N=0$. Under the (weak) assumption $\varlimsup_{m \rightarrow \infty}\left\|\beta q_{m}\right\|>0$ we prove the existence of certain infinite sets $\mathcal{M}_{k} \subseteq \mathbb{N}$ that satisfy $\mathcal{M}_{k} \subseteq \mathcal{M}_{k+1}$ for all positive integers $k, \bigcup_{k=1}^{\infty} \mathcal{M}_{k}=\mathbb{N}$ and

$$
\lim _{N \in \mathcal{M}_{k}} \frac{1}{N} \sum_{k=1}^{N} f(\{k \alpha\})=\int_{0}^{1} f(x) d x .
$$

Under a mild additional assumption $\mathcal{M}_{k+1} \backslash \mathcal{M}_{k}$ can be proved to be infinite for all positive integers $k$.

In the final Section 4 we transfer the results from Section 3 to admissible functions. Many of the proofs in this part are straightforward and will only be sketched.

2. Auxiliary results about the distribution of the sequence $(k \alpha)_{k \geq 1}$. We need some more notation: for any positive integer $N$ we will 
use $\sigma_{N} \in S_{N}$ to denote the uniquely determined permutation such that $\left\{\sigma_{N}(k) \alpha\right\}<\left\{\sigma_{N}(k+1) \alpha\right\}$ for $1 \leq k<N$.

Proposition 2.1. Let $m$ and $b$ be integers, $m \geq 0,1 \leq b \leq a_{m+1}$ and $N=b q_{m}$.

(1) If $m$ is even then

$$
\left\{\sigma_{N}(k) \alpha\right\}= \begin{cases}k\left(q_{m} \alpha-p_{m}\right) & \text { if } 1 \leq k \leq b, \\ \frac{1}{q_{m}}\left\lfloor\frac{k-1}{b}\right\rfloor+\left(q_{m} \alpha-p_{m}\right)\left(b\left\{\frac{k-1}{b}\right\}\right. \\ \left.\quad+\left\{-\frac{q_{m-1}}{q_{m}}\left\lfloor\frac{k-1}{b}\right\rfloor\right\}\right) & \text { if } b<k \leq N .\end{cases}
$$

(2) If $m$ is odd then

$$
\left\{\sigma_{N}(k) \alpha\right\}=\left\{\begin{array}{cl}
-\frac{1}{q_{m}}\left\lfloor-\frac{k}{b}\right\rfloor+\left(q_{m} \alpha-p_{m}\right)\left(b\left\{-\frac{k}{b}\right\}\right. & \\
\left.+\left\{-\frac{q_{m-1}}{q_{m}}\left\lfloor-\frac{k}{b}\right\rfloor\right\}\right) & \text { if } 1 \leq k \leq N-b, \\
1+(N-k+1)\left(q_{m} \alpha-p_{m}\right) & \text { if } N-b<k \leq N .
\end{array}\right.
$$

Proof. This is [1, Proposition 1].

Corollary 2.2. Let $m \geq 0,1 \leq b \leq a_{m+1}$ and $N=b q_{m}$.

(1) If $m$ is even then

$$
\sigma_{N}(k)= \begin{cases}k q_{m} & \text { if } 1 \leq k \leq b, \\ q_{m}\left(b\left\{\frac{k-1}{b}\right\}+\left\{-\frac{q_{m-1}}{q_{m}}\left\lfloor\frac{k-1}{b}\right\rfloor\right\}\right) & \text { if } b<k \leq N .\end{cases}
$$

(2) If $m$ is odd then

$$
\sigma_{N}(k)= \begin{cases}q_{m}\left(b\left\{-\frac{k}{b}\right\}+\left\{-\frac{q_{m-1}}{q_{m}}\left\lfloor-\frac{k}{b}\right\rfloor\right\}\right) & \text { if } 1 \leq k \leq N-b, \\ q_{m}(N-k+1) & \text { if } N-b<k \leq N .\end{cases}
$$

Proof. This follows immediately from Proposition 2.1.

Corollary 2.3. Let $\mu \in(0,1], m \geq 0,1 \leq b \leq \mu a_{m+1}$ and $N=b q_{m}$. If $0 \leq t \leq q_{m}-1$ and $t b+1 \leq k \leq(t+1) b$ then

$$
\left\{\sigma_{N}(k) \alpha\right\} \in \begin{cases}\left(\frac{t}{q_{m}}, \frac{t+\mu}{q_{m}}\right) & \text { if } m \text { is even }, \\ \left(\frac{t+1-\mu}{q_{m}}, \frac{t+1}{q_{m}}\right) & \text { if } m \text { is odd. }\end{cases}
$$


Proof. Let $m$ be even and set $r=k-t b \in\{1, \ldots, b\}$. If $t=0$ then

$$
\left\{\sigma_{N}(k) \alpha\right\}=r\left(q_{m} \alpha-p_{m}\right) \leq b\left(q_{m} \alpha-p_{m}\right) \leq \mu a_{m+1}\left(q_{m} \alpha-p_{m}\right)<\mu / q_{m} .
$$

If $t \geq 1$ then

$$
\begin{aligned}
\frac{t}{q_{m}} & <\left\{\sigma_{N}(k) \alpha\right\}=\frac{t}{q_{m}}+\left(q_{m} \alpha-p_{m}\right)\left(r-1+\left\{-\frac{q_{m-1}}{q_{m}} t\right\}\right) \\
& <\frac{t}{q_{m}}+b\left(q_{m} \alpha-p_{m}\right) \leq \frac{t}{q_{m}}+\mu a_{m+1}\left(q_{m} \alpha-p_{m}\right)<\frac{t+1}{q_{m}} .
\end{aligned}
$$

If $m$ is odd the assertion can be proved analogously.

Corollary 2.4. Let $m \geq 0,1 \leq b \leq a_{m+1}, N=b q_{m}$ and $0 \leq t \leq$ $q_{m}-1$. Then $\left\{\sigma_{N}(k) \alpha\right\} \in\left(t / q_{m},(t+1) / q_{m}\right)$ if and only if $t b+1 \leq k \leq t b+b$. Furthermore, we have

$$
\#\left\{k \mid 1 \leq k \leq N,\{k \alpha\} \in\left(\frac{t}{q_{m}}, \frac{t+1}{q_{m}}\right)\right\}=b .
$$

Proof. Both assertions follow from Corollary 2.3 by setting $\mu=1$.

Lemma 2.5. Let $m \geq 0$.

(1) If $1 \leq N \leq q_{m+1}$ then

$$
\left\{\sigma_{N}(j+1) \alpha\right\}-\left\{\sigma_{N}(j) \alpha\right\} \geq\left|q_{m} \alpha-p_{m}\right| \quad \text { for } 1 \leq j<N .
$$

(2) If $N=q_{m}$ then

$$
\left\{\sigma_{N}(j+1) \alpha\right\}-\left\{\sigma_{N}(j) \alpha\right\} \geq\left|q_{m-1} \alpha-p_{m-1}\right| \quad \text { for } 1 \leq j<N .
$$

Proof. (1) If $1 \leq j<N$ then $\left|\sigma_{N}(j+1)-\sigma_{N}(j)\right|<q_{m+1}$ and therefore

$$
\begin{aligned}
& \left\{\sigma_{N}(j+1) \alpha\right\}-\left\{\sigma_{N}(j) \alpha\right\}=\left|\left\{\sigma_{N}(j+1) \alpha\right\}-\left\{\sigma_{N}(j) \alpha\right\}\right| \\
& \quad=\left|\left(\sigma_{N}(j+1)-\sigma_{N}(j)\right) \alpha-\left\lfloor\sigma_{N}(j+1) \alpha\right\rfloor+\left\lfloor\sigma_{N}(j) \alpha\right\rfloor\right| \geq\left|q_{m} \alpha-p_{m}\right|
\end{aligned}
$$

where we used the fact that convergents are best approximations [18, Chapter II, $\S 3$, Theorem 1].

(2) This is an immediate consequence of (1).

REMARK. Lemma 2.5 can also be derived from stronger results [5].

Lemma 2.6. Let $m \geq 0,1<b \leq a_{m+1}$ and $N=b q_{m}$. If $0 \leq t \leq q_{m}-1$ and $1 \leq r \leq b-1$ then $\left\{\sigma_{N}(t b+r+1) \alpha\right\}-\left\{\sigma_{N}(t b+r) \alpha\right\}=\left|q_{m} \alpha-p_{m}\right|$.

Proof. Let $m$ be even. If $t=0$ the assertion follows immediately from Proposition 2.1. If $t \geq 1$ then 


$$
\begin{aligned}
\left\{\sigma_{N}(t b+r+1) \alpha\right\}-\left\{\sigma_{N}(t b+r) \alpha\right\} & =\frac{t}{q_{m}}+\left(q_{m} \alpha-p_{m}\right)\left(r+\left\{-\frac{q_{m-1}}{q_{m}} t\right\}\right) \\
& \quad-\frac{t}{q_{m}}-\left(q_{m} \alpha-p_{m}\right)\left(r-1+\left\{-\frac{q_{m-1}}{q_{m}} t\right\}\right)=q_{m} \alpha-p_{m} .
\end{aligned}
$$

If $m$ is odd the assertion can be proved analogously.

Lemma 2.7. Let $m \geq 0$ be even, $1 \leq b \leq a_{m+1}$ and $N=b q_{m}$. Then

$$
\left\{\sigma_{N}(t b+1) \alpha\right\}-t / q_{m} \leq\left|q_{m} \alpha-p_{m}\right| \quad \text { for } 0 \leq t \leq q_{m}-1 .
$$

Proof. If $t=0$ the assertion follows immediately from Proposition 2.1. If $t \geq 1$ then

$$
\left\{\sigma_{N}(t b+1) \alpha\right\}-\frac{t}{q_{m}}=\left(q_{m} \alpha-p_{m}\right)\left\{-\frac{q_{m-1}}{q_{m}} t\right\}<q_{m} \alpha-p_{m} .
$$

LEMMA 2.8. Let $m \geq 0,1 \leq b<a_{m+1}$ and $0 \leq t \leq q_{m}-1$.

(1) If $m$ is even then

$$
\left\{\sigma_{(b+1) q_{m}}(t(b+1)+r) \alpha\right\}=\left\{\sigma_{b q_{m}}(t b+r) \alpha\right\} \quad \text { for } 1 \leq r \leq b .
$$

(2) If $m$ is odd then

$$
\left\{\sigma_{(b+1) q_{m}}(t(b+1)+r) \alpha\right\}=\left\{\sigma_{b q_{m}}(t b+r-1) \alpha\right\} \quad \text { for } 2 \leq r \leq b+1 .
$$

Proof. (1) If $t=0$ then

$$
\left\{\sigma_{(b+1) q_{m}}(t(b+1)+r) \alpha\right\}=r\left(q_{m} \alpha-p_{m}\right)=\left\{\sigma_{b q_{m}}(t b+r) \alpha\right\}
$$

for $1 \leq r \leq b$. If $t \geq 1$ then

$$
\begin{aligned}
\left\{\sigma_{(b+1) q_{m}}(t(b+1)+r) \alpha\right\} & =\frac{t}{q_{m}}+\left(q_{m} \alpha-p_{m}\right)\left(r-1+\left\{-\frac{q_{m-1}}{q_{m}} t\right\}\right) \\
& =\left\{\sigma_{b q_{m}}(t b+r) \alpha\right\}
\end{aligned}
$$

for $1 \leq r \leq b$.

(2) This can be proved analogously.

3. Results for functions in $\mathcal{S}_{-}^{+}(\beta)$. We need some more notation. Let $N$ be a positive integer. For $\beta \in(0,1]$ we set

$$
\begin{aligned}
n_{N}^{-} & =\max \left\{k \mid 1 \leq k \leq N,\left\{\sigma_{N}(k) \alpha\right\}<\beta\right\} \\
& =\#\{k \mid 1 \leq k \leq N,\{k \alpha\}<\beta\}
\end{aligned}
$$

and for $\beta \in[0,1)$ we set

$$
\begin{aligned}
n_{N}^{+} & =\min \left\{k \mid 1 \leq k \leq N,\left\{\sigma_{N}(k) \alpha\right\}>\beta\right\} \\
& =N+1-\#\{k \mid 1 \leq k \leq N,\{k \alpha\}>\beta\} .
\end{aligned}
$$


Both quantities are well defined for sufficiently large $N$. Both depend on $\alpha$ and $\beta$ but we usually suppress this in our notation (with the exception of a few lemmata in Section 4). Obviously the sequence $\left(\sigma_{N}\left(n_{N}^{-}\right)\right)_{N \geq 1}$ is increasing and $\lim _{N \rightarrow \infty} \sigma_{N}\left(n_{N}^{-}\right)=\infty$.

Lemma 3.1. Let $f \in \mathcal{S}_{-}^{+}(\beta)$. Then

$$
\lim _{N \rightarrow \infty} \frac{1}{N} f(\{N \alpha\})=0 \Leftrightarrow \lim _{N \rightarrow \infty} \frac{1}{\sigma_{N}\left(n_{N}^{-}\right)} f\left(\left\{\sigma_{N}\left(n_{N}^{-}\right) \alpha\right\}\right)=0 .
$$

Proof. The second condition is necessary because of the remark just before the lemma. It is also sufficient because $\sigma_{N}\left(n_{N}^{-}\right) \leq N$ and $f(\{N \alpha\}) \leq$ $f\left(\left\{\sigma_{N}\left(n_{N}^{-}\right) \alpha\right\}\right)$.

Lemma 3.2. Let $f \in \mathcal{S}_{-}^{+}(\beta), m \geq 0,1 \leq b \leq a_{m+1}$ and $N=b q_{m}$. Then

$$
\frac{1}{N} \sum_{k=1}^{N} f(\{k \alpha\}) \leq 3 \frac{a_{m+1}}{b} \int_{0}^{1} f(x) d x+\frac{1}{\sigma_{N}\left(n_{N}^{-}\right)} f\left(\left\{\sigma_{N}\left(n_{N}^{-}\right) \alpha\right\}\right) .
$$

Proof. Using Lemma 2.5 and the well known inequality

$$
\left|q_{m} \alpha-p_{m}\right|>\frac{1}{\left(a_{m+1}+2\right) q_{m}} \geq \frac{1}{3 a_{m+1} q_{m}}
$$

we can estimate

$$
\begin{aligned}
& \sum_{k=1}^{N} f(\{k \alpha\})=\sum_{k=1}^{n_{N}^{-}} f\left(\left\{\sigma_{N}(k) \alpha\right\}\right) \\
& \quad \leq \sum_{k=1}^{n_{N}^{-}-1} \frac{1}{\left\{\sigma_{N}(k+1) \alpha\right\}-\left\{\sigma_{N}(k) \alpha\right\}} \int_{\left\{\sigma_{N}(k) \alpha\right\}}^{\left\{\sigma_{N}(k+1) \alpha\right\}} f(x) d x+f\left(\left\{\sigma_{N}\left(n_{N}^{-}\right) \alpha\right\}\right) \\
& \quad \leq \frac{1}{\left|q_{m} \alpha-p_{m}\right|} \sum_{k=1}^{n_{N}^{-}-1} \int_{\left\{\sigma_{N}(k) \alpha\right\}} f(x) d x+f\left(\left\{\sigma_{N}\left(n_{N}^{-}\right) \alpha\right\}\right) \\
& \quad \leq 3 a_{m+1} q_{m} \int_{0}^{1} f(x) d x+f\left(\left\{\sigma_{N}\left(n_{N}^{-}\right) \alpha\right\}\right)
\end{aligned}
$$

and therefore

$$
\begin{aligned}
\frac{1}{N} \sum_{k=1}^{N} f(\{k \alpha\}) & \leq 3 \frac{a_{m+1}}{b} \int_{0}^{1} f(x) d x+\frac{1}{N} f\left(\left\{\sigma_{N}\left(n_{N}^{-}\right) \alpha\right\}\right) \\
& \leq 3 \frac{a_{m+1}}{b} \int_{0}^{1} f(x) d x+\frac{1}{\sigma_{N}\left(n_{N}^{-}\right)} f\left(\left\{\sigma_{N}\left(n_{N}^{-}\right) \alpha\right\}\right) .
\end{aligned}
$$


Lemma 3.3. Let $f \in \mathcal{S}_{-}^{+}(\beta), m \geq 0,1 \leq b \leq a_{m+1}$ and $N=b q_{m}$. Then

$$
\begin{aligned}
\sum_{k=1}^{N} f \cdot c_{\left(0,\left(\left\lfloor\beta q_{m}\right\rfloor-1\right) / q_{m}\right)}(\{k \alpha\}) & =\sum_{k=1}^{\left(\left\lfloor\beta q_{m}\right\rfloor-1\right) b} f\left(\left\{\sigma_{N}(k) \alpha\right\}\right) \\
& \leq N \int_{0}^{1} f(x) d x .
\end{aligned}
$$

Proof. The first identity follows from Corollary 2.4, and

$$
\begin{aligned}
& \sum_{k=1}^{\left(\left\lfloor\beta q_{m}\right\rfloor-1\right) b} f\left(\left\{\sigma_{N}(k) \alpha\right\}\right)=\sum_{t=0}^{\left\lfloor\beta q_{m}\right\rfloor-2} \sum_{r=1}^{b} f\left(\left\{\sigma_{N}(t b+r) \alpha\right\}\right) \\
& \leq \sum_{t=0}^{\left\lfloor\beta q_{m}\right\rfloor-2} b f\left(\frac{t+1}{q_{m}}\right) \leq \sum_{t=0}^{\left\lfloor\beta q_{m}\right\rfloor-2} b q_{m} \int_{(t+1) / q_{m}}^{(t+2) / q_{m}} f(x) d x \leq N \int_{0}^{1} f(x) d x .
\end{aligned}
$$

Lemma 3.4. Let $f \in \mathcal{S}_{-}^{+}(\beta), m \geq 0, \mu \in(0,1), 1 \leq b \leq \mu a_{m+1}$ and $N=b q_{m}$. If $m$ is even then

$$
\begin{aligned}
\sum_{k=1}^{N} f \cdot c_{\left(\left(\left\lfloor\beta q_{m}\right\rfloor-1\right) / q_{m},\left\lfloor\beta q_{m}\right\rfloor / q_{m}\right)}(\{k \alpha\}) & =\sum_{k=b\left(\left\lfloor\beta q_{m}\right\rfloor-1\right)+1}^{b\left\lfloor\beta q_{m}\right\rfloor} f\left(\left\{\sigma_{N}(k) \alpha\right\}\right) \\
& \leq \frac{N}{1-\mu} \int_{0}^{1} f(x) d x .
\end{aligned}
$$

Proof. The first identity follows from Corollary 2.4. From Corollary 2.3 we know $\left\{\sigma_{N}(k) \alpha\right\}<\left(\left\lfloor\beta q_{m}\right\rfloor-1+\mu\right) / q_{m}$ for $b\left(\left\lfloor\beta q_{m}\right\rfloor-1\right)+1 \leq k \leq b\left\lfloor\beta q_{m}\right\rfloor$ and therefore

$$
\begin{aligned}
\sum_{k=b\left(\left\lfloor\beta q_{m}\right\rfloor-1\right)+1}^{b\left\lfloor\beta q_{m}\right\rfloor} f\left(\left\{\sigma_{N}(k) \alpha\right\}\right) & \leq b f\left(\left\{\sigma_{N}\left(b\left\lfloor\beta q_{m}\right\rfloor\right) \alpha\right\}\right) \leq b f\left(\frac{\left\lfloor\beta q_{m}\right\rfloor-1+\mu}{q_{m}}\right) \\
\leq & \frac{b q_{m}}{1-\mu} \int_{\left(\left\lfloor\beta q_{m}\right\rfloor-1+\mu\right) / q_{m}}^{\left\lfloor\beta q_{m}\right\rfloor / q_{m}} f(x) d x \leq \frac{N}{1-\mu} \int_{0}^{1} f(x) d x .
\end{aligned}
$$

Lemma 3.5. Let $f \in \mathcal{S}_{-}^{+}(\beta), m \geq 0, \mu \in(0,1), 1 \leq b \leq \mu a_{m+1}$ and $N=b q_{m}$. If $m$ is odd and $n_{N}^{-}>b\left\lfloor\beta q_{m}\right\rfloor$ then

$$
\begin{aligned}
\sum_{k=1}^{N} f \cdot c_{\left(\left(\left\lfloor\beta q_{m}\right\rfloor-1\right) / q_{m},\left\lfloor\beta q_{m}\right\rfloor / q_{m}\right)}(\{k \alpha\}) & =\sum_{k=b\left(\left\lfloor\beta q_{m}\right\rfloor-1\right)+1}^{b\left\lfloor\beta q_{m}\right\rfloor} f\left(\left\{\sigma_{N}(k) \alpha\right\}\right) \\
& \leq \frac{N}{1-\mu} \int_{0}^{1} f(x) d x .
\end{aligned}
$$


Proof. The first identity follows from Corollary 2.4. From Corollary 2.3 we get

$$
\left\{\sigma_{N}(k) \alpha\right\}>\frac{\left\lfloor\beta q_{m}\right\rfloor+1-\mu}{q_{m}} \quad \text { for } b\left\lfloor\beta q_{m}\right\rfloor+1 \leq k \leq b\left\lfloor\beta q_{m}\right\rfloor+b,
$$

which implies $\beta>\left\{\sigma_{N}\left(n_{N}^{-}\right) \alpha\right\}>\left(\left\lfloor\beta q_{m}\right\rfloor+1-\mu\right) / q_{m}$. This yields

$$
\begin{aligned}
\beta-\left\{\sigma_{N}(k) \alpha\right\}>\frac{\left\lfloor\beta q_{m}\right\rfloor+1-\mu}{q_{m}}- & \frac{\left\lfloor\beta q_{m}\right\rfloor}{q_{m}}=\frac{1-\mu}{q_{m}} \\
& \text { for } b\left(\left\lfloor\beta q_{m}\right\rfloor-1\right)+1 \leq k \leq b\left\lfloor\beta q_{m}\right\rfloor .
\end{aligned}
$$

Therefore $\left\{\sigma_{N}(k) \alpha\right\}<\beta-(1-\mu) / q_{m}$ for $b\left(\left\lfloor\beta q_{m}\right\rfloor-1\right)+1 \leq k \leq b\left\lfloor\beta q_{m}\right\rfloor$ and

$$
\begin{aligned}
\sum_{k=b\left(\left\lfloor\beta q_{m}\right\rfloor-1\right)+1}^{b\left\lfloor\beta q_{m}\right\rfloor} f\left(\left\{\sigma_{N}(k) \alpha\right\}\right) & \leq b f\left(\left\{\sigma_{N}\left(b\left\lfloor\beta q_{m}\right\rfloor\right) \alpha\right\}\right) \leq b f\left(\beta-\frac{1-\mu}{q_{m}}\right) \\
& \leq \frac{b q_{m}}{1-\mu} \int_{\beta-(1-\mu) / q_{m}}^{\beta} f(x) d x \leq \frac{N}{1-\mu} \int_{0}^{1} f(x) d x .
\end{aligned}
$$

Lemma 3.6. Let $f \in \mathcal{S}_{-}^{+}(\beta), m \geq 0,1 \leq b \leq a_{m+1}$ and $N=b q_{m}$. If $m$ is odd and $n_{N}^{-}=b\left\lfloor\beta q_{m}\right\rfloor$ then

$$
\frac{1}{N} \sum_{k=1}^{N} f(\{k \alpha\}) \leq \int_{0}^{1} f(x) d x+\frac{1}{\sigma_{N}\left(n_{N}^{-}\right)} f\left(\left\{\sigma_{N}\left(n_{N}^{-}\right) \alpha\right\}\right)
$$

for all sufficiently large $N$.

Proof. Using Lemma 3.3 we get

$$
\begin{aligned}
\sum_{k=1}^{N} f(\{k \alpha\}) & =\sum_{k=1}^{b\left(\left\lfloor\beta q_{m}\right\rfloor-1\right)} f\left(\left\{\sigma_{N}(k) \alpha\right\}\right)+\sum_{k=b\left(\left\lfloor\beta q_{m}\right\rfloor-1\right)+1}^{b\left\lfloor\beta q_{m}\right\rfloor} f\left(\left\{\sigma_{N}(k) \alpha\right\}\right) \\
& \leq N \int_{0}^{1} f(x) d x+b f\left(\left\{\sigma_{N}\left(n_{N}^{-}\right) \alpha\right\}\right)
\end{aligned}
$$

and therefore

$$
\frac{1}{N} \sum_{k=1}^{N} f(\{k \alpha\}) \leq \int_{0}^{1} f(x) d x+\frac{1}{q_{m}} f\left(\left\{\sigma_{N}\left(n_{N}^{-}\right) \alpha\right\}\right) .
$$

If $\beta=1$ then $\sigma_{N}\left(n_{N}^{-}\right)=q_{m}$. If $\beta<1$ then $\sigma_{N}\left(n_{N}^{-}\right)=q_{m}\left\{\frac{q_{m-1}}{q_{m}}\left\lfloor\beta q_{m}\right\rfloor\right\}<q_{m}$ for all sufficiently large $m$. (Here we made use of Corollary 2.2(2) in both cases.) 
Lemma 3.7. Let $f \in \mathcal{S}_{-}^{+}(\beta)$. Then

$$
\frac{1}{q_{m}} \sum_{k=1}^{q_{m}} f(\{k \alpha\}) \leq 3 \int_{0}^{1} f(x) d x+\frac{1}{\sigma_{q_{m}}\left(n_{q_{m}}^{-}\right)} f\left(\left\{\sigma_{q_{m}}\left(n_{q_{m}}^{-}\right) \alpha\right\}\right)
$$

for all sufficiently large $m$.

Proof. We first assume $\beta=1$. If $m$ is odd the assertion follows from Lemma 3.6. If $a_{m+1}=1$ it follows from Lemma 3.2. If $m$ is even and $a_{m+1} \geq 2$ then

$$
\sum_{k=1}^{q_{m}} f \cdot c_{\left(0,1-1 / q_{m}\right)}(\{k \alpha\}) \leq q_{m} \int_{0}^{1} f(x) d x
$$

by Lemma 3.3 and

$$
\sum_{k=1}^{q_{m}} f \cdot c_{\left(1-1 / q_{m}, 1\right)}(\{k \alpha\}) \leq\left(1-\frac{1}{a_{m+1}}\right)^{-1} q_{m} \int_{0}^{1} f(x) d x \leq 2 q_{m} \int_{0}^{1} f(x) d x
$$

by Lemma 3.4. These two inequalities imply the assertion.

Now let $\beta<1$. For sufficiently large $m$ we have $2 / q_{m}<\beta<1-2 / q_{m}$ and we see from Lemma 2.5(2) that

$$
\left\{\sigma_{q_{m}}(j+1) \alpha\right\}-\left\{\sigma_{q_{m}}(j) \alpha\right\} \geq\left|q_{m-1} \alpha-p_{m-1}\right|>\frac{1}{q_{m-1}+q_{m}} \geq \frac{1}{2 q_{m}}
$$

for $1 \leq j<q_{m}$. Estimating as in the proof of Lemma 3.2 we infer

$$
\begin{aligned}
\sum_{k=1}^{q_{m}} f(\{k \alpha\})= & \sum_{k=1}^{n_{q_{m}}^{-}} f\left(\left\{\sigma_{q_{m}}(k) \alpha\right\}\right) \\
\leq & \sum_{k=1}^{n_{q_{m}}^{-}-1} \frac{1}{\left\{\sigma_{q_{m}}(k+1) \alpha\right\}-\left\{\sigma_{q_{m}}(k) \alpha\right\}} \int_{\left\{\sigma_{q_{m}}(k) \alpha\right\}}^{\left\{\sigma_{q_{m}}(k+1) \alpha\right\}} f(x) d x \\
& +f\left(\left\{\sigma_{q_{m}}\left(n_{q_{m}}^{-}\right) \alpha\right\}\right) \\
\leq & 2 q_{m} \int_{0}^{1} f(x) d x+f\left(\left\{\sigma_{q_{m}}\left(n_{q_{m}}^{-}\right) \alpha\right\}\right)
\end{aligned}
$$

and the assertion follows as $\sigma_{q_{m}}\left(n_{q_{m}}^{-}\right) \leq q_{m}$.

We are now able to state our first main result.

Theorem 3.8. Let $f \in \mathcal{S}_{-}^{+}(\beta)$. If $\lim _{N \rightarrow \infty} f(\{N \alpha\}) / N=0$ then

$$
\varliminf_{N \rightarrow \infty} \frac{1}{N} \sum_{k=1}^{N} f(\{k \alpha\})=\lim _{m \rightarrow \infty} \frac{1}{q_{m}} \sum_{k=1}^{q_{m}} f(\{k \alpha\})=\int_{0}^{1} f(x) d x .
$$


Proof. Let $\varepsilon>0$. Choose a $\delta \in(0, \beta)$ such that $\int_{\beta-\delta}^{\beta} f(x) d x<\varepsilon / 3$ and set $f_{\varepsilon}=f \cdot c_{(\beta-\delta, \beta)}$. Then $f_{\varepsilon} \in \mathcal{S}_{-}^{+}(\beta), f_{\varepsilon} \leq f$ and $\int_{0}^{1} f_{\varepsilon}(x) d x<\varepsilon / 3$. As

$$
\begin{aligned}
\varliminf_{N \rightarrow \infty} \frac{1}{N} \sum_{k=1}^{N} f(\{k \alpha\}) & \geq \varliminf_{N \rightarrow \infty} \sum_{k=1}^{N}\left(f-f_{\varepsilon}\right)(\{k \alpha\})=\int_{0}^{1}\left(f-f_{\varepsilon}\right)(x) d x \\
& >\int_{0}^{1} f(x) d x-\varepsilon
\end{aligned}
$$

we can infer

$$
\varliminf_{N \rightarrow \infty} \frac{1}{N} \sum_{k=1}^{N} f(\{k \alpha\}) \geq \int_{0}^{1} f(x) d x,
$$

which implies

$$
\varliminf_{m \rightarrow \infty} \frac{1}{q_{m}} \sum_{k=1}^{q_{m}} f(\{k \alpha\}) \geq \int_{0}^{1} f(x) d x .
$$

On the other hand,

$$
\begin{aligned}
\frac{1}{q_{m}} \sum_{k=1}^{q_{m}} f_{\varepsilon}(\{k \alpha\}) & \leq 3 \int_{0}^{1} f_{\varepsilon}(x) d x+\frac{1}{\sigma_{q_{m}}\left(n_{q_{m}}^{-}\right)} f_{\varepsilon}\left(\left\{\sigma_{q_{m}}\left(n_{q_{m}}^{-}\right) \alpha\right\}\right) \\
& <\varepsilon+\frac{1}{\sigma_{q_{m}}\left(n_{\overline{q_{m}}}^{-}\right)} f\left(\left\{\sigma_{q_{m}}\left(n_{q_{m}}^{-}\right) \alpha\right\}\right)
\end{aligned}
$$

by Lemma 3.7. Therefore

$$
\begin{aligned}
\varlimsup_{m \rightarrow \infty} \frac{1}{q_{m}} \sum_{k=1}^{q_{m}} f(\{k \alpha\}) & =\varlimsup_{m \rightarrow \infty}\left(\frac{1}{q_{m}} \sum_{k=1}^{q_{m}}\left(f-f_{\varepsilon}\right)(\{k \alpha\})+\frac{1}{q_{m}} \sum_{k=1}^{q_{m}} f_{\varepsilon}(\{k \alpha\})\right) \\
& \leq \int_{0}^{1} f(x) d x+\varepsilon .
\end{aligned}
$$

Together with (3.2) this proves

$$
\lim _{m \rightarrow \infty} \frac{1}{q_{m}} \sum_{k=1}^{q_{m}} f(\{k \alpha\})=\int_{0}^{1} f(x) d x
$$

and (3.1) and (3.3) together imply the first equation in the assertion.

REMark. The last theorem is an analogue to equation (1.5) in [3]. It is also interesting to compare Theorem 3.8 with the remark after (1.8) in [3]. Note that what is called $\alpha$ in the present paper is called $\beta$ in [3] and vice versa. 
Lemma 3.9. Let $f \in \mathcal{S}_{-}^{+}(\beta), m \geq 0,1 \leq b \leq a_{m+1}$ and $N=b q_{m}$. Then

$$
\frac{1}{N} \sum_{k=1}^{N} f \cdot c_{\left(0,\left\lfloor\beta q_{m}\right\rfloor / q_{m}\right)}(\{k \alpha\}) \leq 6 \int_{0}^{1} f(x) d x+\frac{1}{\sigma_{N}\left(n_{N}^{-}\right)} f\left(\left\{\sigma_{N}\left(n_{N}^{-}\right) \alpha\right\}\right)
$$

for all sufficiently large $N$.

Proof. If $b \geq a_{m+1} / 2$ this follows from Lemma 3.2. Now let $b<a_{m+1} / 2$ and either let $m$ be even or let $m$ be odd and $n_{N}^{-}>b\left\lfloor\beta q_{m}\right\rfloor$. Then

$$
\begin{aligned}
\frac{1}{N} \sum_{k=1}^{N} f \cdot c_{\left(0,\left\lfloor\beta q_{m}\right\rfloor / q_{m}\right)}(\{k \alpha\})= & \frac{1}{N} \sum_{k=1}^{N} f \cdot c_{\left(0,\left(\left\lfloor\beta q_{m}\right\rfloor-1\right) / q_{m}\right)}(\{k \alpha\}) \\
& +\frac{1}{N} \sum_{k=1}^{N} f \cdot c_{\left(\left(\left\lfloor\beta q_{m}\right\rfloor-1\right) / q_{m},\left\lfloor\beta q_{m}\right\rfloor / q_{m}\right)}(\{k \alpha\}) \\
\leq & \int_{0}^{1} f(x) d x+2 \int_{0}^{1} f(x) d x=3 \int_{0}^{1} f(x) d x
\end{aligned}
$$

where we used Lemma 3.3 and either Lemma 3.4 or Lemma 3.5.

If $b<a_{m+1} / 2,2 \nmid m$ and $n_{N}^{-}=b\left\lfloor\beta q_{m}\right\rfloor$ then

$$
\begin{aligned}
\frac{1}{N} \sum_{k=1}^{N} f \cdot c_{\left(0,\left\lfloor\beta q_{m}\right\rfloor / q_{m}\right)}(\{k \alpha\}) & =\frac{1}{N} \sum_{k=1}^{N} f(\{k \alpha\}) \\
& \leq \int_{0}^{1} f(x) d x+\frac{1}{\sigma_{N}\left(n_{N}^{-}\right)} f\left(\left\{\sigma_{N}\left(n_{N}^{-}\right) \alpha\right\}\right)
\end{aligned}
$$

by Lemma 3.6.

Notations. (1) For $f \in \mathcal{S}_{-}^{+}(\beta)$ and $N \in \mathbb{N}$ we set

$$
S_{N}^{-} f(\beta)=\sum_{k=1}^{N} f \cdot c_{\left(\left\lfloor\beta q_{m}\right\rfloor / q_{m}, \beta\right)}(\{k \alpha\})
$$

where for given $N$ the nonnegative integer $m=m(N)$ is defined via the relation $q_{m} \leq N<q_{m+1}$.

(2) Let $\left(a_{n}\right)_{n \geq 1}$ be a sequence and $\mathcal{N}$ an infinite set of positive integers. If $\mathcal{N}=\left\{n_{k} \mid k \in \mathbb{N}\right\}$ with $n_{k}<n_{k+1}$ for all $k \geq 1$ we set $\lim _{n \in \mathcal{N}} a_{n}=$ $\lim _{k \rightarrow \infty} a_{n_{k}}$ if this limit exists. The quantities $\varlimsup_{n \in \mathcal{N}} a_{n}$ and $\underline{\lim }_{n \in \mathcal{N}} a_{n}$ are defined analogously.

(3) If $\mathcal{J} \subseteq \mathbb{N}$ and $\mu \in(0,1)$ we set $\mathcal{N}_{\mu}(\mathcal{J})=\{n \in \mathbb{N} \mid \exists m \in \mathcal{J}: \mu m \leq$ $n \leq m\}$. 
THEOREM 3.10. Let $f \in \mathcal{S}_{-}^{+}(\beta)$.

(1) $\lim _{N \rightarrow \infty} \frac{1}{N} \sum_{k=1}^{N} f(\{k \alpha\})=\int_{0}^{1} f(x) d x \Rightarrow \lim _{N \rightarrow \infty} \frac{1}{N} f(\{N \alpha\})=0$.

(2) $\lim _{N \in \mathcal{N}} \frac{1}{N} \sum_{k=1}^{N} f(\{k \alpha\})=\int_{0}^{1} f(x) d x \Rightarrow \lim _{N \in \mathcal{N}} \frac{1}{N} S_{N}^{-} f(\beta)=0$.

Proof. Assertion (1) follows trivially from Lemma 1.2 and is stated for future reference and the reader's convenience only. It remains to prove (2). Obviously we have

$$
\begin{aligned}
\varlimsup_{N \in \mathcal{N}} \frac{1}{N} \sum_{k=1}^{N} f \cdot c_{\left(0,\left\lfloor\beta q_{m}\right\rfloor / q_{m}\right)}(\{k \alpha\}) & \leq \lim _{N \in \mathcal{N}} \frac{1}{N} \sum_{k=1}^{N} f(\{k \alpha\}) \\
& =\int_{0}^{1} f(x) d x .
\end{aligned}
$$

Let $\varepsilon>0$. Choose $\delta \in(0, \beta)$ such that $\int_{\beta-\delta}^{\beta} f(x) d x<\varepsilon$ and set $f_{\varepsilon}=$ $f \cdot c_{(\beta-\delta, \beta)}$. Now let $N \in \mathcal{N}$ be sufficiently large such that $\left\lfloor\beta q_{m}\right\rfloor / q_{m}>\beta-\delta$. (We remark that it suffices that $q_{m}>1 / \delta$.) Then

$$
\begin{aligned}
\frac{1}{N} \sum_{k=1}^{N} f \cdot c_{\left(0,\left\lfloor\beta q_{m}\right\rfloor / q_{m}\right)}(\{k \alpha\}) & \geq \frac{1}{N} \sum_{k=1}^{N}\left(f-f_{\varepsilon}\right) \cdot c_{\left(0,\left\lfloor\beta q_{m}\right\rfloor / q_{m}\right)}(\{k \alpha\}) \\
& =\frac{1}{N} \sum_{k=1}^{N}\left(f-f_{\varepsilon}\right)(\{k \alpha\})
\end{aligned}
$$

and therefore

$$
\begin{aligned}
\varliminf_{N \in \mathcal{N}} \frac{1}{N} \sum_{k=1}^{N} f \cdot c_{\left(0,\left\lfloor\beta q_{m}\right\rfloor / q_{m}\right)}(\{k \alpha\}) & \geq \varliminf_{N \in \mathcal{N}} \frac{1}{N} \sum_{k=1}^{N}\left(f-f_{\varepsilon}\right)(\{k \alpha\}) \\
& =\int_{0}^{1}\left(f-f_{\varepsilon}\right)(x) d x>\int_{0}^{1} f(x) d x-\varepsilon .
\end{aligned}
$$

This yields

$$
\varliminf_{N \in \mathcal{N}} \frac{1}{N} \sum_{k=1}^{N} f \cdot c_{\left(0,\left\lfloor\beta q_{m}\right\rfloor / q_{m}\right)}(\{k \alpha\}) \geq \int_{0}^{1} f(x) d x .
$$

The assertion follows from (3.4) and (3.5) together.

Note that $\lim _{N \rightarrow \infty} S_{N}^{-} f(\beta) / N=0$ does not imply $\lim _{N \rightarrow \infty} f(\{N \alpha\}) / N$ $=0$ if there are infinitely many $N$ such that there is no $k \in\{1, \ldots, N\}$ with $\{k \alpha\} \in\left(\left\lfloor\beta q_{m}\right\rfloor / q_{m}, \beta\right)$. 
The following result is a partial inversion of Theorem 3.10. Together with Theorem 3.10 it can be seen as a generalization of Theorem 1.1 for $f \in \mathcal{S}_{-}^{+}(\beta)$ and will prove crucial in what follows.

Theorem 3.11. Let $f \in \mathcal{S}_{-}^{+}(\beta), \mathcal{J} \subseteq\left\{b q_{m} \mid m \geq 0,1 \leq b \leq a_{m+1}\right\}$ be infinite and $\mu \in(0,1)$. If $\lim _{N \rightarrow \infty} f(\{N \alpha\}) / N=0$ and $\lim _{N \in \mathcal{J}} S_{N}^{-} f(\beta) / N$ $=0$ then

$$
\lim _{N \in \mathcal{N}_{\mu}(\mathcal{J})} \frac{1}{N} \sum_{k=1}^{N} f(\{k \alpha\})=\int_{0}^{1} f(x) d x .
$$

Proof. Let $\varepsilon>0$. Choose $\delta \in(0, \beta)$ such that $\int_{\beta-\delta}^{\beta} f(x) d x<\varepsilon \mu / 6$ and set $f_{\varepsilon}=f \cdot c_{(\beta-\delta, \beta)}$. Then $f_{\varepsilon} \in \mathcal{S}_{-}^{+}(\beta), f_{\varepsilon} \leq f$ and $\int_{0}^{1} f_{\varepsilon}(x) d x<\varepsilon \mu / 6$. If $N \in \mathcal{N}_{\mu}(\mathcal{J})$ then there exists a $b q_{m} \in \mathcal{J}$ such that $\mu b q_{m} \leq N \leq b q_{m}$. Employing Lemma 3.9 we get

$$
\begin{aligned}
& \frac{1}{N} \sum_{k=1}^{N} f_{\varepsilon}(\{k \alpha\}) \leq \frac{1}{\mu b q_{m}} \sum_{k=1}^{b q_{m}} f_{\varepsilon}(\{k \alpha\}) \\
& \quad \leq \frac{1}{\mu b q_{m}} \sum_{k=1}^{b q_{m}} f_{\varepsilon} \cdot c_{\left(0,\left\lfloor\beta q_{m}\right\rfloor / q_{m}\right)}(\{k \alpha\})+\frac{1}{\mu b q_{m}} S_{b q_{m}}^{-} f(\beta) \\
& \quad \leq \frac{6}{\mu} \int_{0}^{1} f_{\varepsilon}(x) d x+\frac{1}{\mu \sigma_{b q_{m}}\left(n_{b q_{m}}^{-}\right)} f\left(\left\{\sigma_{b q_{m}}\left(n_{b q_{m}}^{-}\right) \alpha\right\}\right)+\frac{1}{\mu b q_{m}} S_{b q_{m}}^{-} f(\beta) .
\end{aligned}
$$

By our assumptions this leads to

$$
\varlimsup_{N \in \mathcal{N}_{\mu}(\mathcal{J})} \frac{1}{N} \sum_{k=1}^{N} f_{\varepsilon}(\{k \alpha\}) \leq \frac{6}{\mu} \int_{0}^{1} f_{\varepsilon}(x) d x<\varepsilon,
$$

which implies

$$
\begin{aligned}
\varlimsup_{N \in \mathcal{N}_{\mu}(\mathcal{J})} \frac{1}{N} \sum_{k=1}^{N} f(\{k \alpha\}) \\
\quad=\varlimsup_{N \in \mathcal{N}_{\mu}(\mathcal{J})}\left(\frac{1}{N} \sum_{k=1}^{N}\left(f-f_{\varepsilon}\right)(\{k \alpha\})+\frac{1}{N} \sum_{k=1}^{N} f_{\varepsilon}(\{k \alpha\})\right) \\
\quad<\int_{0}^{1} f(x) d x+\varepsilon
\end{aligned}
$$

and therefore

$$
\varlimsup_{N \in \mathcal{N}_{\mu}(\mathcal{J})} \frac{1}{N} \sum_{k=1}^{N} f(\{k \alpha\}) \leq \int_{0}^{1} f(x) d x .
$$


On the other hand,

$$
\varliminf_{N \in \mathcal{N}_{\mu}(\mathcal{J})} \frac{1}{N} \sum_{k=1}^{N} f(\{k \alpha\}) \geq \varliminf_{N \rightarrow \infty} \frac{1}{N} \sum_{k=1}^{N} f(\{k \alpha\})=\int_{0}^{1} f(x) d x
$$

by Theorem 3.8.

In the following corollary we will use the notations $\mathcal{Q}=\left\{q_{m} \mid m \geq 0\right\}$ and $\mathcal{A}_{\mu}=\mathcal{N}_{\mu}(\mathcal{Q})=\left\{N \in \mathbb{N} \mid \exists m \geq 0: \mu q_{m} \leq N \leq q_{m}\right\}$.

Corollary 3.12. Let $f \in \mathcal{S}_{-}^{+}(\beta)$ and $\mu \in(0,1)$. If $\lim _{N \rightarrow \infty} f(\{N \alpha\}) / N$ $=0$ then

$$
\lim _{N \in \mathcal{A}_{\mu}} \frac{1}{N} \sum_{k=1}^{N} f(\{k \alpha\})=\int_{0}^{1} f(x) d x .
$$

Proof. Theorem 3.8 states that

$$
\lim _{N \in \mathcal{Q}} \frac{1}{N} \sum_{k=1}^{N} f(\{k \alpha\})=\int_{0}^{1} f(x) d x .
$$

By Theorem 3.10(2) this implies $\lim _{N \in \mathcal{Q}} S_{N}^{-} f(\beta) / N=0$ and the assertion follows from Theorem 3.11.

Corollary 3.13. Let $f \in \mathcal{S}_{-}^{+} f(\beta)$. If $\alpha$ has bounded continued fraction expansion the following are equivalent:

(1) $\lim _{N \rightarrow \infty} \frac{1}{N} \sum_{k=1}^{N} f(\{k \alpha\})=\int_{0}^{1} f(x) d x, \quad$ (2) $\lim _{N \rightarrow \infty} \frac{1}{N} f(\{N \alpha\})=0$.

Proof. Only the implication $(2) \Rightarrow(1)$ remains to be proved. Let $A=$ $\max _{n \geq 1} a_{n}$ and set $\mu=(A+1)^{-1}$. Then $\mu q_{m} \leq q_{m-1}$ for all $m \geq 1$, that is, we have $\mathcal{A}_{\mu}=\mathbb{N}$. The assertion follows from Corollary 3.12.

Notation. Let $\mathcal{J}$ be an infinite set of nonnegative integers. We set

$$
\mathcal{M}(\mathcal{J})=\left\{N \in \mathbb{N} \mid \exists m \in \mathcal{J}: q_{m} \leq N \leq q_{m+1}\right\} .
$$

Corollary 3.14. Let $f \in \mathcal{S}_{-}^{+}(\beta)$.

(1) If

$$
\lim _{N \rightarrow \infty} \frac{1}{N} \sum_{k=1}^{N} f(\{k \alpha\})=\int_{0}^{1} f(x) d x
$$

then

$$
\lim _{N \rightarrow \infty} \frac{1}{N} f(\{N \alpha\})=0 \quad \text { and } \quad \lim _{m \rightarrow \infty} \max _{1 \leq b \leq a_{m+1}} \frac{1}{b q_{m}} S_{b q_{m}}^{-} f(\beta)=0 .
$$

(2) If

$$
\lim _{N \rightarrow \infty} \frac{1}{N} f(\{N \alpha\})=0 \quad \text { and } \quad \lim _{m \in \mathcal{J}} \max _{1 \leq b \leq a_{m+1}} \frac{1}{b q_{m}} S_{b q_{m}}^{-} f(\beta)=0
$$


then

$$
\lim _{N \in \mathcal{M}(\mathcal{J})} \frac{1}{N} \sum_{k=1}^{N} f(\{k \alpha\})=\int_{0}^{1} f(x) d x .
$$

Proof. (1) This follows from Theorem 3.10.

(2) Let $\widetilde{\mathcal{J}}=\left\{b q_{m} \mid m \in \mathcal{J}, 1 \leq b \leq a_{m+1}\right\}$. We see from the second assumption that $\lim _{N \in \tilde{\mathcal{J}}} S_{N}^{-} f(\beta) / N=0$ and therefore

$$
\lim _{N \in \mathcal{N}_{1 / 2}(\widetilde{\mathcal{J}})} \frac{1}{N} \sum_{k=1}^{N} f(\{k \alpha\})=\int_{0}^{1} f(x) d x
$$

by Theorem 3.11. Furthermore,

$$
\lim _{N \in \mathcal{N}_{1 / 2}(\mathcal{Q})} \frac{1}{N} \sum_{k=1}^{N} f(\{k \alpha\})=\int_{0}^{1} f(x) d x
$$

in view of Corollary 3.12. It is not difficult to check that $\mathcal{M}(\mathcal{J}) \subseteq \mathcal{N}_{1 / 2}(\tilde{\mathcal{J}}) \cup$ $\mathcal{N}_{1 / 2}(\mathcal{Q})$, which completes the proof.

Notation. Let $f \in \mathcal{S}_{-}^{+}(\beta), m \geq 0$ and $1 \leq b \leq a_{m+1}$. We set

$$
\begin{aligned}
& I_{b q_{m}}^{-} f(\beta)=\max \left\{0, \int_{\left\{\sigma_{b q_{m}}\left(b\left\lfloor\beta q_{m}\right\rfloor+1\right) \alpha\right\}}^{\left\{\sigma_{b q_{m}}\left(n_{b q_{m}}^{-}\right) \alpha\right\}} f(x) d x\right\}, \\
& \bar{I}_{b q_{m}}^{-} f(\beta)=\max \left\{0, \int_{\left\lfloor\beta q_{m}\right\rfloor / q_{m}}^{\left\{\sigma_{b q_{m}}\left(n_{b q_{m}}^{-}\right) \alpha\right\}} f(x) d x\right\} .
\end{aligned}
$$

REMARKS. (1) We will not use $\bar{I}_{b q_{m}}^{-}$to establish any results in the remainder of this paper. However, we believe that it will prove useful in the follow-up research. For this reason we include several of its properties and sketch their proofs.

(2) The quantity $I_{b q_{m}}^{-} f(\beta)$ depends on $\alpha$ which usually need not be noted. Only in Section 4 we will occasionally write $I_{b q_{m}(\alpha)}^{-} f(\beta)$ to avoid confusion.

LEMmA 3.15. Let $f \in \mathcal{S}_{-}^{+}(\beta), m \geq 0$ and $1 \leq b \leq a_{m+1}$. Then

$$
\begin{aligned}
\frac{1}{\left|q_{m} \alpha-p_{m}\right|} I_{b q_{m}}^{-} f(\beta) & \leq S_{b q_{m}}^{-} f(\beta) \\
& \leq \frac{1}{\left|q_{m} \alpha-p_{m}\right|} I_{b q_{m}}^{-} f(\beta)+f\left(\left\{\sigma_{b q_{m}}\left(n_{b q_{m}}^{-}\right) \alpha\right\}\right) .
\end{aligned}
$$

If $m$ is even then

$$
\begin{aligned}
\frac{1}{\left|q_{m} \alpha-p_{m}\right|} \bar{I}_{b q_{m}}^{-} f(\beta) & \leq S_{b q_{m}}^{-} f(\beta) \\
& \leq \frac{1}{\left|q_{m} \alpha-p_{m}\right|} \bar{I}_{b q_{m}}^{-} f(\beta)+f\left(\left\{\sigma_{b q_{m}}\left(n_{b q_{m}}^{-}\right) \alpha\right\}\right) .
\end{aligned}
$$


Proof. If there is no $k \in\left\{1, \ldots, b q_{m}\right\}$ such that $\left\lfloor\beta q_{m}\right\rfloor / q_{m}<\{k \alpha\}<\beta$ then $S_{b q_{m}}^{-} f(\beta)=I_{b q_{m}}^{-} f(\beta)=\bar{I}_{b q_{m}}^{-} f(\beta)=0$ and the first assertion is true. Therefore, we may assume from now on that $\left\lfloor\beta q_{m}\right\rfloor / q_{m}<\{k \alpha\}<\beta$ for some $k \in\left\{1, \ldots, b q_{m}\right\}$. Then $n_{N}^{-}=b\left\lfloor\beta q_{m}\right\rfloor+r$ for an $r \in\{1, \ldots, b\}$ by Corollary 2.4 and

$$
S_{b q_{m}}^{-} f(\beta)=\sum_{k=1}^{b q_{m}} f \cdot c_{\left(\left\lfloor\beta q_{m}\right\rfloor / q_{m}, \beta\right)}(\{k \alpha\})=\sum_{j=1}^{r} f\left(\left\{\sigma_{b q_{m}}\left(b\left\lfloor\beta q_{m}\right\rfloor+j\right) \alpha\right\}\right) .
$$

In order to make the remainder of the proof easier to read we introduce the shorthand notation $\omega_{j}=\left\{\sigma_{b q_{m}}\left(b\left\lfloor\beta q_{m}\right\rfloor+j\right) \alpha\right\}$ for $1 \leq j \leq r$. Using Lemma 2.6 we see that

$$
\begin{array}{ll}
\int_{\omega_{j}}^{\omega_{j+1}} f(x) d x \geq\left(\omega_{j+1}-\omega_{j}\right) f\left(\omega_{j}\right)=\left|q_{m} \alpha-p_{m}\right| f\left(\omega_{j}\right) & \text { for } 1 \leq j<r, \\
\omega_{\omega_{j-1}}^{\omega_{j}} f(x) d x \leq\left(\omega_{j}-\omega_{j-1}\right) f\left(\omega_{j}\right)=\left|q_{m} \alpha-p_{m}\right| f\left(\omega_{j}\right) & \text { for } 1<j \leq r .
\end{array}
$$

This implies

$$
\begin{aligned}
f\left(\omega_{1}\right)+ & \frac{1}{\left|q_{m} \alpha-p_{m}\right|} I_{b q_{m}}^{-} f(\beta)=f\left(\omega_{1}\right)+\frac{1}{\left|q_{m} \alpha-p_{m}\right|} \int_{\omega_{1}}^{\omega_{r}} f(x) d x \\
& =f\left(\omega_{1}\right)+\frac{1}{\left|q_{m} \alpha-p_{m}\right|} \sum_{j=2}^{r} \int_{\omega_{j-1}}^{\omega_{j}} f(x) d x \leq f\left(\omega_{1}\right)+\sum_{j=2}^{r} f\left(\omega_{j}\right) \\
& =S_{b q_{m}}^{-} f(\beta)=\sum_{j=1}^{r-1} f\left(\omega_{j}\right)+f\left(\omega_{r}\right) \\
& \leq \frac{1}{\left|q_{m} \alpha-p_{m}\right|} \sum_{j=1}^{r-1} \int_{\omega_{j}}^{\omega_{j+1}} f(x) d x+f\left(\omega_{r}\right) \\
& =\frac{1}{\left|q_{m} \alpha-p_{m}\right|} \int_{\omega_{1}}^{\omega_{r}} f(x) d x+f\left(\omega_{r}\right) \\
& =\frac{1}{\left|q_{m} \alpha-p_{m}\right|} I_{b q_{m}}^{-} f(\beta)+f\left(\omega_{r}\right) .
\end{aligned}
$$

The first assertion follows from $f\left(\omega_{1}\right) \geq 0$. The right-hand inequality of the second assertion follows from $I_{b q_{m}}^{-} f(\beta) \leq \bar{I}_{q_{m}}^{-} f(\beta)$. Using Lemma 2.7 we see 
that

$$
\int_{\left\lfloor\beta q_{m}\right\rfloor / q_{m}}^{\omega_{1}} f(x) d x \leq\left(\omega_{1}-\frac{\left\lfloor\beta q_{m}\right\rfloor}{q_{m}}\right) f\left(\omega_{1}\right) \leq\left|q_{m} \alpha-p_{m}\right| f\left(\omega_{1}\right)
$$

and the left-hand inequality follows from

$$
\int_{\left\lfloor\beta q_{m}\right\rfloor / q_{m}}^{\omega_{1}} f(x) d x+I_{b q_{m}}^{-} f(\beta)=\bar{I}_{b q_{m}}^{-} f(\beta) .
$$

LEMMA 3.16. Let $f \in \mathcal{S}_{-}^{+}(\beta)$ and $\mathcal{J}$ be an infinite set of nonnegative integers. The following conditions are equivalent:

(1) $\lim _{N \rightarrow \infty} \frac{1}{N} f(\{N \alpha\})=0 \quad$ and $\lim _{m \in \mathcal{J}} \max _{1 \leq b \leq a_{m+1}} \frac{1}{b q_{m}} S_{b q_{m}}^{-} f(\beta)=0$,

(2) $\lim _{N \rightarrow \infty} \frac{1}{N} f(\{N \alpha\})=0 \quad$ and $\quad \lim _{m \in \mathcal{J}} \max _{1 \leq b \leq a_{m+1}} \frac{a_{m+1}}{b} I_{b q_{m}}^{-} f(\beta)=0$.

If $\mathcal{J}$ contains only even integers the following condition is equivalent to (1) and (2):

(3) $\lim _{N \rightarrow \infty} \frac{1}{N} f(\{N \alpha\})=0 \quad$ and $\quad \lim _{m \in \mathcal{J}} \max _{1 \leq b \leq a_{m+1}} \frac{a_{m+1}}{b} \bar{I}_{b q_{m}}^{-} f(\beta)=0$.

Proof. For $m \in \mathcal{J}$ we can employ Lemma 3.15 to get

$$
\begin{aligned}
\frac{a_{m+1}}{b} I_{b q_{m}}^{-} f(\beta) & \leq \frac{1}{b q_{m}\left|q_{m} \alpha-p_{m}\right|} I_{b q_{m}}^{-} f(\beta) \leq \frac{1}{b q_{m}} S_{b q_{m}}^{-} f(\beta) \\
& \leq \frac{1}{b q_{m}\left|q_{m} \alpha-p_{m}\right|} I_{b q_{m}}^{-} f(\beta)+\frac{1}{b q_{m}} f\left(\left\{\sigma_{b q_{m}}\left(n_{b q_{m}}^{-}\right) \alpha\right\}\right) \\
& \leq 3 \frac{a_{m+1}}{b} I_{b q_{m}}^{-} f(\beta)+\frac{1}{\sigma_{b q_{m}}\left(n_{b q_{m}}^{-}\right)} f\left(\left\{\sigma_{b q_{m}}\left(n_{b q_{m}}^{-}\right) \alpha\right\}\right) .
\end{aligned}
$$

It is routine to deduce the equivalence of (1) and (2) from this chain of inequalities. The equivalence of (1) and (3) can be proved analogously with the help of the second half of Lemma 3.15.

Corollary 3.17. Let $f \in \mathcal{S}_{-}^{+}(\beta)$.

(1) If

$$
\lim _{N \rightarrow \infty} \frac{1}{N} \sum_{k=1}^{N} f(\{k \alpha\})=\int_{0}^{1} f(x) d x
$$

then $\lim _{N \rightarrow \infty} f(\{N \alpha\}) / N=0$ and

$\lim _{m \rightarrow \infty} \max _{1 \leq b \leq a_{m+1}} \frac{a_{m+1}}{b} I_{b q_{m}}^{-} f(\beta)=0, \quad \lim _{m \rightarrow \infty} \max _{1 \leq b \leq a_{2 m+1}} \frac{a_{2 m+1}}{b} \bar{I}_{b q_{2 m}} f(\beta)=0$. 
(2) Let $\mathcal{J}$ be an infinite set of nonnegative integers. If

$$
\lim _{N \rightarrow \infty} \frac{1}{N} f(\{N \alpha\})=0 \quad \text { and } \quad \lim _{m \in \mathcal{J}} \max _{1 \leq b \leq a_{m+1}} \frac{a_{m+1}}{b} I_{b q_{m}}^{-} f(\beta)=0
$$

then

$$
\lim _{N \in \mathcal{M}(\mathcal{J})} \frac{1}{N} \sum_{k=1}^{N} f(\{k \alpha\})=\int_{0}^{1} f(x) d x .
$$

(3) Let $\mathcal{J}$ be an infinite set of even nonnegative integers. If

$$
\lim _{N \rightarrow \infty} \frac{1}{N} f(\{N \alpha\})=0 \quad \text { and } \quad \lim _{m \in \mathcal{J}} \max _{1 \leq b \leq a_{m+1}} \frac{a_{m+1}}{b} \bar{I}_{b q_{m}}^{-} f(\beta)=0
$$

then

$$
\lim _{N \in \mathcal{M}(\mathcal{J})} \frac{1}{N} \sum_{k=1}^{N} f(\{k \alpha\})=\int_{0}^{1} f(x) d x .
$$

Proof. All assertions can be deduced immediately from Corollary 3.14 and Lemma 3.16.

LEMMA 3.18. Let $m \geq 0$ be sufficiently large, $1 \leq b \leq a_{m+1}$ and $\beta \in$ $(0,1)$. Furthermore, assume the existence of a $k \in\left\{1, \ldots, b q_{m}\right\}$ such that $\{k \alpha\} \in\left(\left\lfloor\beta q_{m}\right\rfloor / q_{m}, \beta\right)$.

If $m$ is even the following two conditions are equivalent:

(1) $\left\{\sigma_{b q_{m}}\left(b\left\lfloor\beta q_{m}\right\rfloor+b\right) \alpha\right\}<\beta$,

(2) $b<\left\{\beta q_{m}\right\}\left[a_{m+1}, a_{m+2}, \ldots\right]+1-\left\{-\beta q_{m-1}\right\}+\delta_{m}$ where $\delta_{m}=$ $\left\lfloor\left\{-\beta q_{m-1}\right\}+\frac{q_{m-1}}{q_{m}}\left\{\beta q_{m}\right\}\right\rfloor \in\{0,1\}$.

If $m$ is odd the following two conditions are equivalent:

(1) $\left\{\sigma_{b q_{m}}\left(b\left\lfloor\beta q_{m}\right\rfloor+1\right) \alpha\right\}<\beta$,

(2) $b>\left[a_{m+1}, a_{m+2}, \ldots\right]\left(1-\left\{\beta q_{m}\right\}\right)+1-\left\{\beta q_{m-1}\right\}+\delta_{m}$ where $\delta_{m}=$ $\left\lfloor\left\{\beta q_{m-1}\right\}+\frac{q_{m-1}}{q_{m}}\left(1-\left\{\beta q_{m}\right\}\right)\right\rfloor \in\{0,1\}$.

Proof. As $\beta \in(0,1)$ we can assume $2 / q_{m}<\beta<1-2 / q_{m}$. Let $m$ be even. By Proposition 2.1(1) we find the following:

$$
\begin{aligned}
\left\{\sigma_{b q_{m}}\left(b\left\lfloor\beta q_{m}\right\rfloor+b\right) \alpha\right\}<\beta & \\
& \Leftrightarrow \frac{\left\lfloor\beta q_{m}\right\rfloor}{q_{m}}+\left(q_{m} \alpha-p_{m}\right)\left(b-1+\left\{-\frac{q_{m-1}}{q_{m}}\left\lfloor\beta q_{m}\right\rfloor\right\}\right)<\frac{\beta q_{m}}{q_{m}} \\
& \Leftrightarrow\left(q_{m} \alpha-p_{m}\right)\left(b-1+\left\{\left\{-\beta q_{m-1}\right\}+\frac{q_{m-1}}{q_{m}}\left\{\beta q_{m}\right\}\right\}\right)<\frac{\left\{\beta q_{m}\right\}}{q_{m}}
\end{aligned}
$$




$$
\begin{gathered}
\Leftrightarrow b-1+\left\{-\beta q_{m-1}\right\}+\left[0, a_{m}, \ldots, a_{1}\right]\left\{\beta q_{m}\right\}-\delta_{m}<\frac{\left\{\beta q_{m}\right\}}{q_{m}\left(q_{m} \alpha-p_{m}\right)} \\
=\left(\left[a_{m+1}, a_{m+2}, \ldots\right]+\left[0, a_{m}, \ldots, a_{1}\right]\right)\left\{\beta q_{m}\right\} \\
\Leftrightarrow b<\left[a_{m+1}, a_{m+2}, \ldots\right]\left\{\beta q_{m}\right\}+1-\left\{-\beta q_{m-1}\right\}+\delta_{m} .
\end{gathered}
$$

The assertion for odd $m$ can be proved analogously.

LemMA 3.19. Let $f \in \mathcal{S}_{-}^{+}(\beta)$ and $m \geq 0$ even. Set

$$
\mathcal{B}_{m}=\left\{b \mid 1 \leq b \leq a_{m+1},\left\{\sigma_{b q_{m}}\left(b\left\lfloor\beta q_{m}\right\rfloor+b\right) \alpha\right\}<\beta\right\} .
$$

Then the two maps $\mathcal{B}_{m} \rightarrow \mathbb{R}$ given by $b \mapsto b^{-1} I_{b q_{m}}^{-} f(\beta)$ and $b \mapsto b^{-1} \bar{I}_{b q_{m}}^{-} f(\beta)$ are both increasing for sufficiently large $m$.

Proof. We see from Corollary 2.4 and Lemma 2.8 that

$$
\begin{aligned}
\frac{\left\lfloor\beta q_{m}\right\rfloor}{q_{m}} & <\left\{\sigma_{q_{m}}\left(\left\lfloor\beta q_{m}\right\rfloor+1\right) \alpha\right\}<\left\{\sigma_{2 q_{m}}\left(2\left\lfloor\beta q_{m}\right\rfloor+2\right) \alpha\right\}<\cdots \\
& <\left\{\sigma_{a_{m+1} q_{m}}\left(a_{m+1}\left\lfloor\beta q_{m}\right\rfloor+a_{m+1}\right) \alpha\right\}<\frac{\left\lfloor\beta q_{m}\right\rfloor+1}{q_{m}}
\end{aligned}
$$

and therefore $\mathcal{B}_{m}=\emptyset$ if and only if $\beta \leq\left\{\sigma_{q_{m}}\left(\left\lfloor\beta q_{m}\right\rfloor+1\right) \alpha\right\}$. If, on the other hand, $\mathcal{B}_{m} \neq \emptyset$ there exists a $b_{0}(m) \in\left\{1, \ldots, a_{m+1}\right\}$ such that $\mathcal{B}_{m}=$ $\left\{1, \ldots, b_{0}(m)\right\}$. Our aim is to prove that

$$
\frac{1}{b} I_{b q_{m}}^{-} f(\beta) \leq \frac{1}{b+1} I_{(b+1) q_{m}}^{-} f(\beta) \quad \text { for } 1 \leq b<b_{0}(m) .
$$

Similar to the proof of Lemma 3.15 we will use the shorthand notations

$$
\begin{aligned}
\omega_{j} & =\left\{\sigma_{b q_{m}}\left(b\left\lfloor\beta q_{m}\right\rfloor+j\right) \alpha\right\} \\
& =\left\{\sigma_{(b+1) q_{m}}\left((b+1)\left\lfloor\beta q_{m}\right\rfloor+j\right) \alpha\right\} \quad \text { for } 1 \leq j \leq b
\end{aligned}
$$

(where we used Lemma 2.8(1)) and

$$
\omega_{b+1}=\left\{\sigma_{(b+1) q_{m}}\left((b+1)\left\lfloor\beta q_{m}\right\rfloor+b+1\right) \alpha\right\} .
$$

Then

$$
\begin{aligned}
\frac{1}{b} I_{b q_{m}}^{-} f(\beta) & \leq \frac{1}{b+1} I_{(b+1) q_{m}}^{-} f(\beta) \\
& \Leftrightarrow(b+1) \int_{\omega_{1}}^{\omega_{b}} f(x) d x \leq b \int_{\omega_{1}}^{\omega_{b+1}} f(x) d x \\
& \Leftrightarrow b \int_{\omega_{1}}^{\omega_{b}} f(x) d x+\int_{\omega_{1}}^{\omega_{b}} f(x) d x \leq b \int_{\omega_{1}}^{\omega_{b}} f(x) d x+b \int_{\omega_{b}}^{\omega_{b+1}} f(x) d x \\
& \Leftrightarrow \sum_{j=1}^{b-1} \int_{\omega_{j}}^{\omega_{j+1}} f(x) d x \leq b \int_{\omega_{b}}^{\omega_{b+1}} f(x) d x
\end{aligned}
$$


and the assertion follows as $\omega_{j+1}-\omega_{j}=q_{m} \alpha-p_{m}$ for $1 \leq j \leq b$ (by Lemma $2.6)$. The second assertion's proof is largely analogous. The only additional ingredient we need is the fact that

$$
\int_{\left\lfloor\beta q_{m}\right\rfloor / q_{m}}^{\omega_{1}} f(x) d x \leq \int_{\omega_{b}}^{\omega_{b+1}} f(x) d x
$$

which follows from Lemma 2.7 .

LEMma 3.20. Let $f \in \mathcal{S}_{-}^{+}(\beta)$ and $m \geq 0$.

(1) If $\beta q_{m} \in \mathbb{N}$ then

$$
\max _{1 \leq b \leq a_{m+1}} \frac{a_{m+1}}{b} I_{b q_{m}}^{-} f(\beta)=0
$$

and

$$
\max _{1 \leq b \leq a_{m+1}} \frac{a_{m+1}}{b} \bar{I}_{b q_{m}}^{-} f(\beta)=0 \quad \text { if } m \text { is even. }
$$

(2) If $\beta q_{m} \notin \mathbb{N}$ then

$$
\max _{1 \leq b \leq a_{m+1}} \frac{a_{m+1}}{b} I_{b q_{m}}^{-} f(\beta) \leq \frac{2}{\left\|\beta q_{m}\right\|} \int_{\left\lfloor\beta q_{m}\right\rfloor / q_{m}}^{\beta} f(x) d x
$$

and

$$
\begin{aligned}
\max _{1 \leq b \leq a_{m+1}} \frac{a_{m+1}}{b} I_{b q_{m}}^{-} f(\beta) & \leq \max _{1 \leq b \leq a_{m+1}} \frac{a_{m+1}}{b} \bar{I}_{b q_{m}}^{-} f(\beta) \\
& \leq \frac{2}{\left\|\beta q_{m}\right\|} \int_{\left\lfloor\beta q_{m}\right\rfloor / q_{m}}^{\beta} f(x) d x \quad \text { if } m \text { is even } .
\end{aligned}
$$

Proof. (1) If $\beta q_{m} \in \mathbb{N}$ then $I_{b q_{m}}^{-} f(\beta)=0$ (and $\bar{I}_{b q_{m}}^{-} f(\beta)=0$ if $m$ is even) for $1 \leq b \leq a_{m+1}$.

(2) Let $m$ be even. The left-hand inequality follows from $I_{b q_{m}}^{-} f(\beta) \leq$ $\bar{I}_{b q_{m}}^{-} f(\beta)$ for $1 \leq b \leq a_{m+1}$. If there is no $k \in\left\{1, \ldots, a_{m+1} q_{m}\right\}$ such that $\{k \alpha\} \in\left(\left\lfloor\beta q_{m}\right\rfloor / q_{m}, \beta\right)$ then $\bar{I}_{b q_{m}}^{-} f(\beta)=0$ for $1 \leq b \leq a_{m+1}$ and the righthand inequality is trivial. So we may assume from now on that such a $k$ exist. Lemma 3.19 implies

$$
\max _{1 \leq b \leq a_{m+1}} \frac{a_{m+1}}{b} \bar{I}_{b q_{m}}^{-} f(\beta)=\frac{a_{m+1}}{b_{0}(m)} \bar{I}_{b_{0}(m) q_{m}}^{-} f(\beta) .
$$

(Here $b_{0}(m)$ has the same meaning as in the proof of Lemma 3.19.) Note that $\sigma_{b q_{m}}\left(n_{b q_{m}}^{-}\right)=\sigma_{b_{0}(m) q_{m}}\left(n_{b_{0}(m) q_{m}}^{-}\right)$and therefore $\bar{I}_{b q_{m}}^{-} f(\beta)=\bar{I}_{b_{0}(m) q_{m}}^{-} f(\beta)$ for $b>b_{0}(m)$. On account of Lemma 3.18,

$$
b_{0}(m)<\left\{\beta q_{m}\right\}\left[a_{m+1}, a_{m+2}, \ldots\right]+1-\left\{-\beta q_{m-1}\right\}+\delta_{m} \leq b_{0}(m)+1
$$


and thus $b_{0}(m)>a_{m+1}\left\{\beta q_{m}\right\}-1$. This implies $a_{m+1}\left\{\beta q_{m}\right\}<2 b_{0}(m)$. Therefore, we have

$$
\frac{a_{m+1}}{b_{0}(m)} \bar{I}_{b_{0}(m) q_{m}}^{-} f(\beta) \leq \frac{2}{\left\{\beta q_{m}\right\}} \int_{\left\lfloor\beta q_{m}\right\rfloor / q_{m}}^{\beta} f(x) d x .
$$

We now assume that $m$ is odd. If there is no $k \in\left\{1, \ldots, a_{m+1} q_{m}\right\}$ such that $\{k \alpha\} \in\left(\left\lfloor\beta q_{m}\right\rfloor / q_{m}, \beta\right)$ then $I_{b q_{m}}^{-} f(\beta)=0$ for $1 \leq b \leq a_{m+1}$ and the assertion is trivial. If there is such a $k \in\left\{1, \ldots, b q_{m}\right\}$ then $\left\{\sigma_{b q_{m}}\left(b\left\lfloor\beta q_{m}\right\rfloor+1\right) \alpha\right\}<\beta$ and

$$
\begin{aligned}
b & > \\
& \left.>a_{m+1}, a_{m+2}, \ldots\right]\left(1-\left\{\beta q_{m}\right\}\right)+1-\left\{\beta q_{m-1}\right\}+\delta_{m} \\
& a_{m+1}\left(1-\left\{\beta q_{m}\right\}\right) .
\end{aligned}
$$

(If, on the other hand,

$$
b \leq\left[a_{m+1}, a_{m+2}, \ldots\right]\left(1-\left\{\beta q_{m}\right\}\right)+1-\left\{\beta q_{m-1}\right\}+\delta_{m}
$$

then $I_{b q_{m}}^{-} f(\beta)=0$.) Therefore, we get

$$
\max _{1 \leq b \leq a_{m+1}} \frac{a_{m+1}}{b} I_{b q_{m}}^{-} f(\beta)<\frac{1}{1-\left\{\beta q_{m}\right\}} \int_{\left\lfloor\beta q_{m}\right\rfloor / q_{m}}^{\beta} f(x) d x .
$$

Theorem 3.21. Let $f \in \mathcal{S}_{-}^{+} f(\beta)$. If $\underline{\lim }_{m \rightarrow \infty}\left\|\beta q_{m}\right\|>0$ or $\beta \in \mathbb{Q}$ the following two conditions are equivalent:

$$
\text { (1) } \lim _{N \rightarrow \infty} \frac{1}{N} \sum_{k=1}^{N} f(\{k \alpha\})=\int_{0}^{1} f(x) d x, \quad \text { (2) } \lim _{N \rightarrow \infty} \frac{1}{N} f(\{N \alpha\})=0 .
$$

Proof. Only the implication $(2) \Rightarrow(1)$ needs to be proved. Assume that $\mu=\underline{\lim }_{m \rightarrow \infty}\left\|\beta q_{m}\right\|>0$. Then $\left\|\beta q_{m}\right\| \geq \mu / 2$ for all sufficiently large $m$ and

$$
\frac{1}{\left\|\beta q_{m}\right\|} \int_{\left\lfloor\beta q_{m}\right\rfloor / q_{m}}^{\beta} f(x) d x \leq \frac{2}{\mu} \int_{\left\lfloor\beta q_{m}\right\rfloor / q_{m}}^{\beta} f(x) d x \rightarrow 0
$$

as $m \rightarrow \infty$. The assertion follows from Lemma 3.20 and Corollary 3.17(2). Now let $\beta \in \mathbb{Q}$. Let $\mathcal{J}_{1}=\left\{m \geq 0 \mid \beta q_{m} \in \mathbb{N}\right\}$ and $\mathcal{J}_{2}=\left\{m \geq 0 \mid \beta q_{m} \notin \mathbb{N}\right\}$. If $m \in \mathcal{J}_{1}$ then $I_{b q_{m}}^{-} f(\beta)=0$ for $1 \leq b \leq a_{m+1}$ and trivially

$$
\lim _{m \in \mathcal{J}_{1}} \max _{1 \leq b \leq a_{m+1}} \frac{a_{m+1}}{b} I_{b q_{m}}^{-} f(\beta)=0 .
$$

Assume that $\beta=p / q$ where $p, q$ are positive, coprime integers. If $m \in \mathcal{J}_{2}$ then $\left\|\beta q_{m}\right\| \geq 1 / q$, which leads to

$$
\lim _{m \in \mathcal{J}_{2}} \max _{1 \leq b \leq a_{m+1}} \frac{a_{m+1}}{b} I_{b q_{m}}^{-} f(\beta)=0
$$

just as in the case $\underline{\lim }_{m \rightarrow \infty}\left\|\beta q_{m}\right\|>0$. As $\mathcal{J}_{1} \cup \mathcal{J}_{2}=\mathbb{N} \cup\{0\}$ the assertion follows from Corollary 3.17(2). 
Theorem 3.22. Let $\beta \notin \mathbb{Q}, f \in \mathcal{S}_{-}^{+}(\beta), \lim _{N \rightarrow \infty} f(\{N \alpha\}) / N=0$ and $\mu=\overline{\lim }_{m \rightarrow \infty}\left\|\beta q_{m}\right\|>0$. For all positive integers $k$ we set $\mathcal{J}_{k}=\{m \in$ $\left.\mathbb{N} \cup\{0\} \mid\left\|\beta q_{m}\right\|>\mu /(k+1)\right\}$ and $\mathcal{M}_{k}=\mathcal{M}\left(\mathcal{J}_{k}\right)$. The sets $\mathcal{M}_{k}$ are all infinite and have the following properties:

(1) $\mathcal{M}_{k} \subseteq \mathcal{M}_{k+1}$ for all $k \geq 1$.

(2) $\bigcup_{k=1}^{\infty} \mathcal{M}_{k}=\mathbb{N}$.

(3) $\lim _{N \in \mathcal{M}_{k}} N^{-1} \sum_{k=1}^{N} f(\{k \alpha\})=\int_{0}^{1} f(x) d x$ for all $k \geq 1$.

(4) If $\left(\beta q_{m}\right)_{m \geq 0}$ is uniformly distributed modulo 1 then $\mathcal{M}_{k+1} \backslash \mathcal{M}_{k}$ is infinite for all $k \geq 1$.

Proof. It is trivial that $\mathcal{M}_{k}$ is infinite for all $k \geq 1$.

(1) Obviously $\mathcal{J}_{k} \subseteq \mathcal{J}_{k+1}$, which implies $\mathcal{M}_{k} \subseteq \mathcal{M}_{k+1}$ for $k \geq 1$.

(2) For $N \in \mathbb{N}$ choose $m \geq 0$ such that $q_{m} \leq N<q_{m+1}$. There is a positive integer $k$ such that $\left\|\beta q_{m}\right\|>\mu /(k+1)$, which means that $m \in \mathcal{J}_{k}$ and $N \in \mathcal{M}_{k}$.

(3) If $m \in \mathcal{J}_{k}$ then

$$
\max _{1 \leq b \leq a_{m+1}} \frac{a_{m+1}}{b} I_{b q_{m}}^{-} f(\beta) \ll \frac{k+1}{\mu} \int_{\left\lfloor\beta q_{m}\right\rfloor / q_{m}}^{\beta} f(x) d x
$$

by Lemma 3.20, and the assertion follows from Corollary 3.17(2).

(4) For any positive integer $k$ there are infinitely many nonnegative $m$ such that

$$
\frac{\mu}{k+2}<\left\{\beta q_{m}\right\}=\left\|\beta q_{m}\right\| \leq \frac{\mu}{k+1}
$$

or equivalently $m \in \mathcal{J}_{k+1} \backslash \mathcal{J}_{k}$. For each such $m$ we choose a positive integer $N$ with $q_{m}<N<q_{m+1}$. Then $N \in \mathcal{M}_{k+1} \backslash \mathcal{M}_{k}$.

REMARK. For fixed irrational $\alpha$ the sequence $\left(\beta q_{m}\right)_{m \geq 0}$ is uniformly distributed modulo 1 for almost all $\beta$ [12, Chapter 1, Theorem 4.1]. This implies that $\underline{\lim }_{m \rightarrow \infty}\left\|\beta q_{m}\right\|=0$ and $\varlimsup_{m \rightarrow \infty}\left\|\beta q_{m}\right\|=1 / 2$ for almost all $\beta$. As a consequence we see that Theorem 3.22 is a result about the average case. The only exceptional case we had to exclude is $\lim _{m \rightarrow \infty}\left\|\beta q_{m}\right\|=0$. The set of all $\beta$ with this property has Lebesgue measure zero and was studied in detail in $[13,11]$.

4. Results for admissible functions. In this final section we will transfer the results from Section 3 to admissible functions. We remind the reader that such functions have a representation

$$
f=f_{0}+f_{1}^{-}+\cdots+f_{r}^{-}+f_{1}^{+}+\cdots+f_{s}^{+}
$$

with $f_{0}$ Riemann-integrable, $f_{i}^{-} \in \mathcal{S}_{-}^{+}\left(\beta_{i}^{-}\right) \cup \mathcal{S}_{-}^{-}\left(\beta_{i}^{-}\right)$for $1 \leq i \leq r$ and $f_{j}^{+} \in$ $\mathcal{S}_{+}^{+}\left(\beta_{j}^{+}\right) \cup \mathcal{S}_{+}^{-}\left(\beta_{j}^{+}\right)$for $1 \leq j \leq s$. We will keep these notations throughout this section. 
Proposition 4.1. If $\alpha \in \mathbb{R} \backslash \mathbb{Q}$ has continued fraction expansion $\alpha=$ $\left[a_{0}, a_{1}, a_{2}, \ldots\right]$ then $-\alpha$ has continued fraction expansion

$$
-\alpha= \begin{cases}{\left[-a_{0}-1,1, a_{1}-1, a_{2}, a_{3}, \ldots\right]} & \text { if } a_{1}>1 \\ {\left[-a_{0}-1, a_{2}+1, a_{3}, a_{4}, \ldots\right]} & \text { if } a_{1}=1 .\end{cases}
$$

Proof. This fact can be found, for example, in O. Perron's well known textbook [17].

Corollary 4.2. For irrational $\alpha$ we have

and

$$
q_{m}(-\alpha)= \begin{cases}q_{m-1}(\alpha) & \text { for all } m \geq 1 \text { if } a_{1}(\alpha)>1, \\ q_{m+1}(\alpha) & \text { for all } m \geq 0 \text { if } a_{1}(\alpha)=1,\end{cases}
$$

$$
p_{m}(-\alpha)= \begin{cases}-p_{m-1}(\alpha) & \text { for all } m \geq 1 \text { if } a_{1}(\alpha)>1 \\ -p_{m+1}(\alpha) & \text { for all } m \geq 0 \text { if } a_{1}(\alpha)=1\end{cases}
$$

Proof. This follows from Proposition 4.1 by induction.

Remark. As a consequence of Proposition 4.1 and Corollary 4.2 we have

$$
a_{m+1}(\alpha)= \begin{cases}a_{m+2}(-\alpha) & \text { for all } m \geq 1 \text { if } a_{1}(\alpha)>1\left(\Leftrightarrow a_{1}(-\alpha)=1\right), \\ a_{m}(-\alpha) & \text { for all } m \geq 2 \text { if } a_{1}(\alpha)=1\left(\Leftrightarrow a_{1}(-\alpha)>1\right),\end{cases}
$$

and

$$
q_{m}(\alpha)= \begin{cases}q_{m+1}(-\alpha) & \text { for all } m \geq 0 \text { if } a_{1}(\alpha)>1\left(\Leftrightarrow a_{1}(-\alpha)=1\right), \\ q_{m-1}(-\alpha) & \text { for all } m \geq 1 \text { if } a_{1}(\alpha)=1\left(\Leftrightarrow a_{1}(-\alpha)>1\right) .\end{cases}
$$

We will usually apply Proposition 4.1 and Corollary 4.2 in this form and will simply write $q_{m}(\alpha)=q_{m \pm 1}(-\alpha)$ without repeating that

$$
\pm 1= \begin{cases}+1 & \text { if } a_{1}(\alpha)>1 \\ -1 & \text { if } a_{1}(\alpha)=1\end{cases}
$$

Lemma 4.3. Let $f$ be admissible. If $\lim _{N \rightarrow \infty} f(\{N \alpha\}) / N=0$ then $\lim _{N \rightarrow \infty} f_{i}^{-}(\{N \alpha\}) / N=0$ for $1 \leq i \leq r$ and $\lim _{N \rightarrow \infty} f_{j}^{+}(\{N \alpha\}) / N=0$ for $1 \leq j \leq s$.

Proof. Let $1 \leq i \leq r$. There is an $\varepsilon>0$ such that $f_{1}^{-}, \ldots, f_{i-1}^{-}, f_{i+1}^{-}, \ldots$ $\ldots, f_{r}^{-}, f_{1}^{+}, \ldots, f_{s}^{+}$are all bounded on the interval $\left(\beta_{i}^{-}-\varepsilon, \beta_{i}^{-}\right)$and $f_{i}^{-}$is bounded on $\left[0, \beta_{i}^{-}-\varepsilon\right] \cup\left[\beta_{i}^{-}, 1\right]$. Let $\mathcal{N}_{1}=\left\{N \in \mathbb{N} \mid\{N \alpha\} \in\left(\beta_{i}^{-}-\varepsilon, \beta_{i}^{-}\right)\right\}$ and $\mathcal{N}_{2}=\mathbb{N} \backslash \mathcal{N}_{1}$. As the sequence $\left(f_{i}^{-}(\{N \alpha\})\right)_{N \in \mathcal{N}_{2}}$ is bounded we get $\lim _{N \in \mathcal{N}_{2}} f_{i}^{-}(\{N \alpha\}) / N=0$.

On the other hand, the sequence $\left(\left(f-f_{i}^{-}\right)(\{N \alpha\})\right)_{N \in \mathcal{N}_{1}}$ is bounded and therefore

$$
\lim _{N \in \mathcal{N}_{1}} \frac{1}{N} f_{i}^{-}(\{N \alpha\})=\lim _{N \in \mathcal{N}_{1}}\left(\frac{1}{N} f(\{N \alpha\})-\frac{1}{N}\left(f-f_{i}^{-}\right)(\{N \alpha\})\right)=0 .
$$

The second assertion can be proved analogously. 
THEOREM 4.4. Let $f$ be admissible.

(1) If $\lim _{N \rightarrow \infty} f(\{N \alpha\}) / N=0$ then

$$
\lim _{m \rightarrow \infty} \frac{1}{q_{m}} \sum_{k=1}^{q_{m}} f(\{k \alpha\})=\int_{0}^{1} f(x) d x .
$$

(2) If in addition $f_{i}^{-} \in \mathcal{S}_{-}^{+}\left(\beta_{i}^{-}\right)$for $1 \leq i \leq r$ and $f_{j}^{+} \in \mathcal{S}_{+}^{+}\left(\beta_{j}^{+}\right)$for $1 \leq j \leq s$ then

$$
\varliminf_{N \rightarrow \infty} \frac{1}{N} \sum_{k=1}^{N} f(\{k \alpha\})=\int_{0}^{1} f(x) d x .
$$

Alternatively, if $f_{i}^{-} \in \mathcal{S}_{-}^{-}\left(\beta_{i}^{-}\right)$for $1 \leq i \leq r$ and $f_{j}^{+} \in \mathcal{S}_{+}^{-}\left(\beta_{j}^{+}\right)$for $1 \leq j \leq s$ then

$$
\varlimsup_{N \rightarrow \infty} \frac{1}{N} \sum_{k=1}^{N} f(\{k \alpha\})=\int_{0}^{1} f(x) d x .
$$

Proof. (1) This has been proved in Theorem 3.8 for $f \in \mathcal{S}_{-}^{+}(\beta)$ for some $\beta \in(0,1]$. If $f \in \mathcal{S}_{-}^{-}(\beta)$ then $-f \in \mathcal{S}_{-}^{+}(\beta)$. As $\lim _{N \rightarrow \infty}(-f(\{N \alpha\})) / N=0$ Theorem 3.8 implies

$$
\begin{aligned}
\lim _{m \rightarrow \infty} \frac{1}{q_{m}} \sum_{k=1}^{q_{m}} f(\{k \alpha\}) & =-\lim _{m \rightarrow \infty} \frac{1}{q_{m}} \sum_{k=1}^{q_{m}}(-f(\{k \alpha\})) \\
& =-\int_{0}^{1}(-f(x)) d x=\int_{0}^{1} f(x) d x .
\end{aligned}
$$

Let now $f \in \mathcal{S}_{+}^{+}(\beta)$ or $f \in \mathcal{S}_{+}^{-}(\beta)$ for some $\beta \in[0,1)$. Then $\widehat{f} \in \mathcal{S}_{-}^{+}(1-\beta)$ or $\widehat{f} \in \mathcal{S}_{-}^{-}(1-\beta)$ and $\lim _{N \rightarrow \infty} \widehat{f}(\{N(-\alpha)\}) / N=\lim _{N \rightarrow \infty} f(\{N \alpha\}) / N=0$. Therefore

$$
\begin{aligned}
\lim _{m \rightarrow \infty} \frac{1}{q_{m}(\alpha)} \sum_{k=1}^{q_{m}(\alpha)} f(\{k \alpha\}) & =\lim _{m \rightarrow \infty} \frac{1}{q_{m \pm 1}(-\alpha)} \sum_{k=1}^{q_{m \pm 1}(-\alpha)} \widehat{f}(\{k(-\alpha)\}) \\
& =\int_{0}^{1} \widehat{f}(x) d x=\int_{0}^{1} f(x) d x .
\end{aligned}
$$

Let now $f$ be admissible. Then

$$
\begin{array}{ll}
\lim _{N \rightarrow \infty} \frac{1}{N} f_{i}^{-}(\{N \alpha\})=0 & \text { for } 1 \leq i \leq r, \\
\lim _{N \rightarrow \infty} \frac{1}{N} f_{j}^{+}(\{N \alpha\})=0 & \text { for } 1 \leq j \leq s
\end{array}
$$


by Lemma 4.3. Using the special cases we proved so far we find

$$
\begin{aligned}
\lim _{m \rightarrow \infty} \frac{1}{q_{m}} \sum_{k=1}^{q_{m}} f(\{k \alpha\}) & \\
= & \lim _{m \rightarrow \infty} \frac{1}{q_{m}} \sum_{k=1}^{q_{m}} f_{0}(\{k \alpha\})+\sum_{i=1}^{r} \lim _{m \rightarrow \infty} \frac{1}{q_{m}} \sum_{k=1}^{q_{m}} f_{i}^{-}(\{k \alpha\}) \\
& +\sum_{j=1}^{s} \lim _{m \rightarrow \infty} \frac{1}{q_{m}} \sum_{k=1}^{q_{m}} f_{j}^{+}(\{k \alpha\}) \\
= & \int_{0}^{1} f_{0}(x) d x+\sum_{i=1}^{r} \int_{0}^{1} f_{i}^{-}(x) d x+\sum_{j=1}^{s} \int_{0}^{1} f_{j}^{+}(x) d x=\int_{0}^{1} f(x) d x .
\end{aligned}
$$

(2) This can be proved as (1) with minimal changes, that is, starting from Theorem 3.8 we first transfer the result to $f \in \mathcal{S}_{-}^{-}(\beta)$, then to functions in $\mathcal{S}_{+}^{+}(\beta)$ and $\mathcal{S}_{+}^{-}(\beta)$, and finally to general admissible functions.

Remark. The method of proof we employed in Theorem 4.4, that is, to transfer a result about $f \in \mathcal{S}_{-}^{+}(\beta)$ to a result about admissible functions, will be used several times in the remainder of Section 4 . As this is a rather mechanical process we will usually not give all the details.

ThEOREM 4.5. Let $f$ be admissible such that $\lim _{N \rightarrow \infty} f(\{N \alpha\}) / N=0$, $\mu \in(0,1)$ and $\mathcal{A}_{\mu}$ as in Corollary 3.12. Then

$$
\lim _{N \in \mathcal{A}_{\mu}} \frac{1}{N} \sum_{k=1}^{N} f(\{k \alpha\})=\int_{0}^{1} f(x) d x .
$$

Proof. This can be proved by the procedure described in the proof of Theorem 4.4 starting from Corollary 3.12. Note that $\mathcal{A}_{\mu}=\{N \in \mathbb{N} \mid \exists m \geq$ $\left.0: \mu q_{m}(\alpha) \leq N \leq q_{m}(\alpha)\right\}$ and if we set $\widehat{\mathcal{A}}_{\mu}=\{N \in \mathbb{N} \mid \exists m \geq 0$ : $\left.\mu q_{m}(-\alpha) \leq N \leq q_{m}(-\alpha)\right\}$ then $\mathcal{A}_{\mu}=\widehat{\mathcal{A}}_{\mu}$.

THEOREM 4.6. Let $f$ be admissible. If $\alpha$ has bounded continued fraction expansion the following two conditions are equivalent:

(1) $\lim _{N \rightarrow \infty} \frac{1}{N} \sum_{k=1}^{N} f(\{k \alpha\})=\int_{0}^{1} f(x) d x, \quad$ (2) $\lim _{N \rightarrow \infty} \frac{1}{N} f(\{N \alpha\})=0$.

Proof. By Proposition 4.1 the continued fraction expansion of $-\alpha$ is bounded if and only if the continued fraction expansion of $\alpha$ is bounded. Using this fact we can deduce the implication $(2) \Rightarrow(1)$ from Corollary 3.13 by the procedure described in the proof of Theorem 4.4 . 
Theorem 4.7. Let $f$ be admissible. If $\underline{\lim }_{m \rightarrow \infty}\left\|\beta_{i}^{-} q_{m}\right\|>0$ or $\beta_{i}^{-} \in \mathbb{Q}$ for $1 \leq i \leq r$, and $\underline{\lim }_{m \rightarrow \infty}\left\|\beta_{j}^{+} q_{m}\right\|>0$ or $\beta_{j}^{+} \in \mathbb{Q}$ for $1 \leq j \leq s$, the following two conditions are equivalent:

(1) $\lim _{N \rightarrow \infty} \frac{1}{N} \sum_{k=1}^{N} f(\{k \alpha\})=\int_{0}^{1} f(x) d x, \quad$ (2) $\lim _{N \rightarrow \infty} \frac{1}{N} f(\{N \alpha\})=0$.

Proof. If $\underline{\lim }_{m \rightarrow \infty}\left\|\beta q_{m}\right\|>0$ then

$$
\varliminf_{m \rightarrow \infty}\left\|(1-\beta) q_{m}(-\alpha)\right\|=\varliminf_{m \rightarrow \infty}\left\|\beta q_{m \pm 1}(\alpha)\right\|=\varliminf_{m \rightarrow \infty}\left\|\beta q_{m}(\alpha)\right\|>0 .
$$

Using this and the trivial fact that $1-\beta \in \mathbb{Q}$ if and only if $\beta \in \mathbb{Q}$ we can deduce the implication $(2) \Rightarrow(1)$ from Theorem 3.21 , again by the procedure described in the proof of Theorem 4.4.

Notation. As a counterpart to the permutation $\sigma_{N} \in S_{N}$ introduced in Section 2 we now define $\tau_{N} \in S_{N}$ to be the uniquely determined permutation such that $\left\{\tau_{N}(k)(-\alpha)\right\}<\left\{\tau_{N}(k+1)(-\alpha)\right\}$ for $1 \leq k<N$.

Lemma 4.8. Let $N$ be a positive integer. Then

(1) $\tau_{N}(k)=\sigma_{N}(N-k+1)$ for $1 \leq k \leq N$,

(2) $\left\{\tau_{N}(k)(-\alpha)\right\}=1-\left\{\sigma_{N}(N-k+1) \alpha\right\}$ for $1 \leq k \leq N$,

(3) $n_{N}^{-}(-\alpha, 1-\beta)=N+1-n_{N}^{+}(\alpha, \beta)$ and $n_{N}^{+}(-\alpha, 1-\beta)=N+1-$ $n_{N}^{-}(\alpha, \beta)$.

Proof. All three properties can be deduced from the various definitions within a few lines. We remind the reader that the definitions of $n_{N}^{-}$and $n_{N}^{+}$ can be found at the beginning of Section 3 .

Notation. Let $m \geq 0$ and $1 \leq b \leq a_{m+1}$. We now extend the definitions of $I_{b q_{m}}^{-} f(\beta)$ and $\bar{I}_{b q_{m}}^{-} f(\beta)$. If $f \in \mathcal{S}_{-}^{+}(\beta)$ or $f \in \mathcal{S}_{-}^{-}(\beta)$ for some $\beta \in(0,1]$ then

$$
\begin{aligned}
& I_{b q_{m}}^{-} f(\beta)=\max \left\{0, \int_{\left\{\sigma_{b q_{m}}\left(b\left\lfloor\beta q_{m}\right\rfloor+1\right) \alpha\right\}}^{\left\{\sigma_{b q_{m}}\left(n_{b q_{m}}^{-}\right) \alpha\right\}}|f(x)| d x\right\}, \\
& \bar{I}_{b q_{m}}^{-} f(\beta)=\max \left\{0, \int_{\left\lfloor\beta q_{m}\right\rfloor / q_{m}}^{\left\{\sigma_{b q_{m}}\left(n_{b q_{m}}^{-}\right) \alpha\right\}}|f(x)| d x\right\} .
\end{aligned}
$$

If $f \in \mathcal{S}_{+}^{+}(\beta)$ or $f \in \mathcal{S}_{+}^{-}(\beta)$ for some $\beta \in[0,1)$ we define

$$
I_{b q_{m}}^{+} f(\beta)=\max \left\{0, \int_{\left\{\sigma_{b q_{m}}\left(n_{b q_{m}}^{+}\right) \alpha\right\}}^{\left\{\sigma_{b q_{m}}\left(b\left\lceil\beta q_{m}\right\rceil\right) \alpha\right\}}|f(x)| d x\right\},
$$




$$
\bar{I}_{b q_{m}}^{+} f(\beta)=\max \left\{0, \int_{\left\{\sigma_{b q_{m}}\left(n_{b q_{m}}^{+}\right) \alpha\right\}}^{\left\lceil\beta q_{m}\right\rceil / q_{m}}|f(x)| d x\right\}
$$

If it is necessary to stress the dependence on $\alpha$ we write $I_{b q_{m}(\alpha)}^{-} f(\beta)$, etc.

LEMMA 4.9. (1) Let $m \geq 0$ and $1 \leq b \leq a_{m+1}$. If $f \in \mathcal{S}_{-}^{-} f(\beta)$ for some $\beta \in(0,1]$ then $I_{b q_{m}}^{-} f(\beta)=I_{b q_{m}}^{-}(-f)(\beta)$ and $\bar{I}_{b q_{m}}^{-} f(\beta)=\bar{I}_{b q_{m}}^{-}(-f)(\beta)$.

(2) Let $m \geq 2$ and $1 \leq b \leq a_{m+1}$. If $f \in \mathcal{S}_{+}^{+}(\beta)$ or $f \in \mathcal{S}_{+}^{-}(\beta)$ for some $\beta \in[0,1)$ then $I_{b q_{m}(\alpha)}^{+} f(\beta)=I_{b q_{m \pm 1}(-\alpha)}^{-} \widehat{f}(1-\beta)$ and $\bar{I}_{b q_{m}(\alpha)}^{+} f(\beta)=$ $\bar{I}_{b q_{m \pm 1}(-\alpha)}^{-} \hat{f}(1-\beta)$.

Proof. (1) This follows trivially from the definition.

(2) It follows from Lemma 4.8(2), (3) that

$$
\begin{aligned}
\left\{\tau_{b q_{m \pm 1}(-\alpha)}\left(n_{b q_{m \pm 1}(-\alpha)}^{-}(-\alpha, 1-\beta)\right)(-\alpha)\right\} & \\
& =1-\left\{\sigma_{b q_{m}(\alpha)}\left(n_{b q_{m}(\alpha)}^{+}(\alpha, \beta)\right) \alpha\right\} .
\end{aligned}
$$

Using Lemma 4.8(2) one can deduce within a few lines that

$$
\begin{aligned}
\left\{\tau _ { b q _ { m \pm 1 } ( - \alpha ) } \left(b\left\lfloor(1-\beta) q_{m \pm 1}(-\alpha)\right\rfloor\right.\right. & +1)(-\alpha)\} \\
& =1-\left\{\sigma_{b q_{m}(\alpha)}\left(b\left\lceil\beta q_{m}(\alpha)\right\rceil\right) \alpha\right\} .
\end{aligned}
$$

In addition one can check that

$$
\left\lfloor(1-\beta) q_{m \pm 1}(-\alpha)\right\rfloor / q_{m \pm 1}(-\alpha)=1-\left\lceil\beta q_{m}(\alpha)\right\rceil / q_{m}(\alpha) .
$$

Note that (4.2) and (4.3) are correct both if $\beta q_{m} \in \mathbb{N}$ and $\beta q_{m} \notin \mathbb{N}$. By using (4.1)-(4.3) the two equations in (2) can be deduced from the definitions given just before Lemma 4.9 .

Notation. Let $I^{+}=\left\{i \mid 1 \leq i \leq r, f_{i}^{-} \in \mathcal{S}_{-}^{+}\left(\beta_{i}^{-}\right)\right\}, I^{-}=\{i \mid 1 \leq i \leq r$, $\left.f_{i}^{-} \in \mathcal{S}_{-}^{-}\left(\beta_{i}^{-}\right)\right\}, J^{+}=\left\{j \mid 1 \leq j \leq s, f_{j}^{+} \in \mathcal{S}_{+}^{+}\left(\beta_{j}^{+}\right)\right\}$and $J^{-}=\{j \mid 1 \leq j$ $\left.\leq s, f_{j}^{+} \in \mathcal{S}_{+}^{-}\left(\beta_{j}^{+}\right)\right\}$.

THEOREM 4.10. Let $f$ be admissible.

(1) If

$$
\lim _{N \rightarrow \infty} \frac{1}{N} \sum_{k=1}^{N}\left(\sum_{i \in I^{+}} f_{i}^{-}+\sum_{j \in J^{+}} f_{j}^{+}\right)(\{k \alpha\})=\int_{0}^{1}\left(\sum_{i \in I^{+}} f_{i}^{-}+\sum_{j \in J^{+}} f_{j}^{+}\right)(x) d x
$$

and

$$
\lim _{N \rightarrow \infty} \frac{1}{N} \sum_{k=1}^{N}\left(\sum_{i \in I^{-}} f_{i}^{-}+\sum_{j \in J^{-}} f_{j}^{+}\right)(\{k \alpha\})=\int_{0}^{1}\left(\sum_{i \in I^{-}} f_{i}^{-}+\sum_{j \in J^{-}} f_{j}^{+}\right)(x) d x
$$


then

$$
\begin{gathered}
\lim _{N \rightarrow \infty} \frac{1}{N} f_{i}^{-}(\{N \alpha\})=0 \quad \text { for } 1 \leq i \leq r, \\
\lim _{N \rightarrow \infty} \frac{1}{N} f_{j}^{+}(\{N \alpha\})=0 \quad \text { for } 1 \leq j \leq s, \\
\lim _{m \rightarrow \infty} \max _{1 \leq b \leq a_{m+1}} \frac{a_{m+1}}{b} I_{b q_{m}}^{-} f_{i}^{-}\left(\beta_{i}^{-}\right)=0 \quad \text { for } 1 \leq i \leq r, \\
\lim _{m \rightarrow \infty} \max _{1 \leq b \leq a_{m+1}} \frac{a_{m+1}}{b} I_{b q_{m}}^{+} f_{j}^{+}\left(\beta_{j}^{+}\right)=0 \quad \text { for } 1 \leq j \leq s, \\
\lim _{m \rightarrow \infty} \max _{1 \leq b \leq a_{2 m+1}} \frac{a_{2 m+1}}{b} \bar{I}_{b q_{2 m}}^{-} f_{i}^{-}\left(\beta_{i}^{-}\right)=0 \quad \text { for } 1 \leq i \leq r, \\
\lim _{m \rightarrow \infty} \max _{1 \leq b \leq a_{2 m}} \frac{a_{2 m}}{b} \bar{I}_{b q_{2 m-1}}^{+} f_{j}^{+}\left(\beta_{j}^{+}\right)=0 \quad \text { for } 1 \leq j \leq s .
\end{gathered}
$$

(2) Let $\lim _{N \rightarrow \infty} f(\{N \alpha\}) / N=0$. If $\mathcal{J}$ is an infinite set of nonnegative even integers and

$$
\begin{aligned}
& \lim _{m \in \mathcal{J}} \max _{1 \leq b \leq a_{m+1}} \frac{a_{m+1}}{b} I_{b q_{m}}^{-} f_{i}^{-}\left(\beta_{i}^{-}\right)=0 \quad \text { for } 1 \leq i \leq r, \\
& \lim _{m \in \mathcal{J}} \max _{1 \leq b \leq a_{m+1}} \frac{a_{m+1}}{b} I_{b q_{m}}^{+} f_{j}^{+}\left(\beta_{j}^{+}\right)=0 \quad \text { for } 1 \leq j \leq s,
\end{aligned}
$$

then

$$
\lim _{N \in \mathcal{M}(\mathcal{J})} \frac{1}{N} \sum_{k=1}^{N} f(\{k \alpha\})=\int_{0}^{1} f(x) d x .
$$

(3) Let $\lim _{N \rightarrow \infty} f(\{N \alpha\}) / N=0$. If $\mathcal{J}$ is an infinite set of nonnegative even integers and

then

$$
\lim _{m \in \mathcal{J}} \max _{1 \leq b \leq a_{m+1}} \frac{a_{m+1}}{b} \bar{I}_{b q_{m}}^{-} f_{i}^{-}\left(\beta_{i}^{-}\right)=0
$$

$$
\lim _{N \in \mathcal{M}(\mathcal{J})} \frac{1}{N} \sum_{k=1}^{N} f_{i}^{-}(\{k \alpha\})=\int_{0}^{1} f_{i}^{-}(x) d x
$$

for $1 \leq i \leq r$. If $\mathcal{J}$ is an infinite set of odd positive integers and

then

$$
\lim _{m \in \mathcal{J}} \max _{1 \leq b \leq a_{m+1}} \frac{a_{m+1}}{b} \bar{I}_{b q_{m}}^{+} f_{j}^{+}\left(\beta_{j}^{+}\right)=0
$$

$$
\lim _{N \in \mathcal{M}(\mathcal{J})} \frac{1}{N} \sum_{k=1}^{N} f_{j}^{+}(\{k \alpha\})=\int_{0}^{1} f_{j}^{+}(x) d x
$$

for $1 \leq j \leq s$.

Proof. (1) It follows from (4.4) and (4.5) that

$$
\lim _{N \rightarrow \infty} \frac{1}{N} \sum_{k=1}^{N} f(\{k \alpha\})=\int_{0}^{1} f(x) d x .
$$


This implies $\lim _{N \rightarrow \infty} f(\{N \alpha\}) / N=0$ from which we get (4.6) by Lemma 4.3. Assertion (4.7) has been proved for $f \in \mathcal{S}_{-}^{+}(\beta)$ in Corollary 3.17(1). Using Lemma $4.9(1)$ it can be proved for $f \in \mathcal{S}_{-}^{-}(\beta)$ and with the help of Corollary 4.9(2) we get (4.8) for $f \in \mathcal{S}_{+}^{+}(\beta)$ and $f \in \mathcal{S}_{+}^{-}(\beta)$. Let now $f$ be admissible. By Theorem 4.4,

$$
\begin{array}{ll}
\varliminf_{N \rightarrow \infty} \frac{1}{N} \sum_{k=1}^{N} f_{i}^{-}(\{k \alpha\})=\int_{0}^{1} f_{i}^{-}(x) d x & \text { for } i \in I^{+}, \\
\varliminf_{N \rightarrow \infty} \frac{1}{N} \sum_{k=1}^{N} f_{j}^{+}(\{k \alpha\})=\int_{0}^{1} f_{j}^{+}(x) d x & \text { for } j \in J^{+} .
\end{array}
$$

By (4.4) the last two identities yield

$$
\begin{array}{ll}
\lim _{N \rightarrow \infty} \frac{1}{N} \sum_{k=1}^{N} f_{i}^{-}(\{k \alpha\})=\int_{0}^{1} f_{i}^{-}(x) d x & \text { for } i \in I^{+}, \\
\lim _{N \rightarrow \infty} \frac{1}{N} \sum_{k=1}^{N} f_{j}^{+}(\{k \alpha\})=\int_{0}^{1} f_{j}^{+}(x) d x & \text { for } j \in J^{+},
\end{array}
$$

which imply (4.7) for $i \in I^{+}$and (4.8) for $j \in J^{+}$. (The last but one step is the reason we made assumptions (4.4) and (4.5), which are stronger than what one might expect.) It requires only minimal changes to prove (4.7) for $i \in I^{-}$and (4.8) for $j \in J^{-}$. The identities (4.9) and (4.10) can be proved along the same lines.

$(2,3)$ Both can be deduced from Corollary 3.17 by the standard procedure we first employed in the proof of Theorem 4.4. Note that if we set

$$
\widehat{\mathcal{J}}=\{m \pm 1 \mid m \in \mathcal{J}\}= \begin{cases}\{m+1 \mid m \in \mathcal{J}\} & \text { if } a_{1}(\alpha)>1, \\ \{m-1 \mid m \in \mathcal{J}\} & \text { if } a_{1}(\alpha)=1,\end{cases}
$$

then $\mathcal{M}(\widehat{\mathcal{J}})=\mathcal{M}(\mathcal{J})$.

Lemma 4.11. (1) Let $f \in \mathcal{S}_{-}^{+}(\beta)$ or $f \in \mathcal{S}_{-}^{-}(\beta)$ for some $\beta \in(0,1]$ and $m \geq 0$. If $\beta q_{m} \in \mathbb{N}$ then

$$
\max _{1 \leq b \leq a_{m+1}} \frac{a_{m+1}}{b} I_{b q_{m}}^{-} f(\beta)=0
$$

and

$$
\max _{1 \leq b \leq a_{m+1}} \frac{a_{m+1}}{b} \bar{I}_{b q_{m}}^{-} f(\beta)=0 \quad \text { if } m \text { is even. }
$$

If $\beta q_{m} \notin \mathbb{N}$ then

$$
\max _{1 \leq b \leq a_{m+1}} \frac{a_{m+1}}{b} I_{b q_{m}}^{-} f(\beta) \leq \frac{2}{\left\|\beta q_{m}\right\|} \int_{\left\lfloor\beta q_{m}\right\rfloor / q_{m}}^{\beta}|f(x)| d x
$$


and

$$
\begin{aligned}
\max _{1 \leq b \leq a_{m+1}} \frac{a_{m+1}}{b} I_{b q_{m}}^{-} f(\beta) & \leq \max _{1 \leq b \leq a_{m+1}} \frac{a_{m+1}}{b} \bar{I}_{b q_{m}}^{-} f(\beta) \\
& \leq \frac{2}{\left\|\beta q_{m}\right\|} \int_{\left\lfloor\beta q_{m}\right\rfloor / q_{m}}^{\beta}|f(x)| d x \quad \text { if } m \text { is even } .
\end{aligned}
$$

(2) Let $f \in \mathcal{S}_{+}^{+}(\beta)$ or $f \in \mathcal{S}_{+}^{-}(\beta)$ for some $\beta \in[0,1)$ and $m \geq 2$. If $\beta q_{m} \in \mathbb{N}$ then

$$
\max _{1 \leq b \leq a_{m+1}} \frac{a_{m+1}}{b} I_{b q_{m}}^{+} f(\beta)=0
$$

and

$$
\max _{1 \leq b \leq a_{m+1}} \frac{a_{m+1}}{b} \bar{I}_{b q_{m}}^{+} f(\beta)=0 \quad \text { if } m \text { is odd. }
$$

If $\beta q_{m} \notin \mathbb{N}$ then

$$
\max _{1 \leq b \leq a_{m+1}} \frac{a_{m+1}}{b} I_{b q_{m}}^{+} f(\beta) \leq \frac{2}{\left\|\beta q_{m}\right\|} \int_{\beta}^{\left\lceil\beta q_{m}\right\rceil / q_{m}}|f(x)| d x
$$

and

$$
\begin{aligned}
\max _{1 \leq b \leq a_{m+1}} \frac{a_{m+1}}{b} I_{b q_{m}}^{+} f(\beta) & \leq \max _{1 \leq b \leq a_{m+1}} \frac{a_{m+1}}{b} \bar{I}_{b q_{m}}^{+} f(\beta) \\
& \leq \frac{2}{\left\|\beta q_{m}\right\|} \int_{\beta}^{\left\lceil\beta q_{m}\right\rceil / q_{m}}|f(x)| d x \quad \text { if } m \text { is odd } .
\end{aligned}
$$

Proof. (1) This has been proved in Lemma 3.20 for $f \in \mathcal{S}_{-}^{+}(\beta)$ and follows from Lemma 4.9(1) for $f \in \mathcal{S}_{-}^{-}(\beta)$.

(2) This can be reduced to (1) with the help of Lemma 4.9(2). Note that

$$
\left\|(1-\beta) q_{m \pm 1}(-\alpha)\right\|=\left\|\beta q_{m}(\alpha)\right\|, \quad \frac{\left\lfloor(1-\beta) q_{m \pm 1}(-\alpha)\right\rfloor}{q_{m \pm 1}(-\alpha)}=1-\frac{\left\lceil\beta q_{m}(\alpha)\right\rceil}{q_{m}(\alpha)} \text {. }
$$

Notation. Let $f$ be admissible. We set $B_{m}=\min \left(\left\{\left\|\beta_{i}^{-} q_{m}\right\| \mid 1 \leq i \leq r, \beta_{i}^{-} \notin \mathbb{Q}\right\} \cup\left\{\left\|\beta_{j}^{+} q_{m}\right\| \mid 1 \leq j \leq s, \beta_{j}^{+} \notin \mathbb{Q}\right\}\right)$.

Theorem 4.12. Let $f$ be admissible, $\left\{\beta_{1}^{-}, \ldots, \beta_{r}^{-}, \beta_{1}^{+}, \ldots, \beta_{s}^{+}\right\} \backslash \mathbb{Q} \neq \emptyset$, and

$$
\lim _{N \rightarrow \infty} \frac{1}{N} f(\{N \alpha\})=0, \quad \mu=\varlimsup_{m \rightarrow \infty} B_{m}>0 .
$$

For all positive integers $k$ we set $\mathcal{J}_{k}=\left\{m \in \mathbb{N} \cup\{0\} \mid B_{m}>\mu /(k+1)\right\}$ and $\mathcal{M}_{k}=\mathcal{M}\left(\mathcal{J}_{k}\right)$. The sets $\mathcal{M}_{k}$ are all infinite and have the following properties:

(1) $\mathcal{M}_{k} \subseteq \mathcal{M}_{k+1}$ for all $k \geq 1$.

(2) $\bigcup_{k=1}^{\infty} \mathcal{M}_{k}=\mathbb{N}$. 
(3) $\lim _{N \in \mathcal{M}_{k}} N^{-1} \sum_{k=1}^{N} f(\{k \alpha\})=\int_{0}^{1} f(x) d x$ for all $k \geq 1$.

(4) Let $\left\{i_{1}, \ldots, i_{\varrho}\right\}=\left\{i \mid 1 \leq i \leq r, \beta_{i}^{-} \notin \mathbb{Q}\right\}$ and $\left\{j_{1}, \ldots, j_{\sigma}\right\}=\{j \mid$ $\left.1 \leq j \leq s, \beta_{j}^{+} \notin \mathbb{Q}\right\}$. If $\left(\beta_{i_{1}}^{-} q_{m}, \ldots, \beta_{i_{\rho}}^{-} q_{m}, \beta_{j_{1}}^{+} q_{m}, \ldots, \beta_{j_{\sigma}}^{+} q_{m}\right)_{m \geq 0}$ is uniformly distributed modulo 1 in $\mathbb{R}^{\varrho+\sigma}$ then $\mathcal{M}_{k+1} \backslash \mathcal{M}_{k}$ is infinite for all $k \geq 1$.

Proof. There are hardly any changes compared with the proof of Theorem 3.22 in the proofs of (1) and (2). Theorems 4.7 and 4.10(2) are used to prove (3). For the proof of (4) note that there are infinitely many $m$ such that

$$
\left(\left\{\beta_{i_{1}}^{-} q_{m}\right\}, \ldots,\left\{\beta_{i_{\varrho}}^{-} q_{m}\right\},\left\{\beta_{j_{1}}^{+} q_{m}\right\}, \ldots,\left\{\beta_{j_{\sigma}}^{+} q_{m}\right\}\right) \in\left(\frac{\mu}{k+2}, \frac{\mu}{k+1}\right]^{\varrho+\sigma},
$$

which implies $B_{m} \in(\mu /(k+2), \mu /(k+1)]$. From this point on the proof is analogous to that of Theorem 3.22(4).

Remarks. (1) For fixed irrational $\alpha$ the sequence $\left(\beta_{1} q_{m}, \ldots, \beta_{\tau} q_{m}\right)_{m \geq 0}$ is uniformly distributed modulo 1 for almost all $\left(\beta_{1}, \ldots, \beta_{\tau}\right)[12$, Chapter 1 , Exercise 6.12]. This shows that Theorem 4.12 again describes the average case.

(2) If $\beta_{1}, \ldots, \beta_{\tau} \in[0,1] \backslash \mathbb{Q}$ and $\left(\beta_{1} q_{m}, \ldots, \beta_{\tau} q_{m}\right)_{m \geq 0}$ is uniformly distributed modulo 1 then $\varlimsup_{m \rightarrow \infty} \min \left\{\left\|\beta_{1} q_{m}\right\|, \ldots,\left\|\beta_{\tau} q_{m}\right\|\right\}=1 / 2$.

(3) A very interesting question we do not answer in this paper is whether an analogue of Theorem 1.1 is true for arbitrary $F$ or whether Theorems 3.22 and 4.12 are best possible. We believe that it is unlikely that the complete analogue is true but so far we have not been successful in constructing a counterexample. However, we hope to return to this question in a future paper.

Acknowledgements. The major part of this paper was written while the author was an Erwin Schrödinger Fellow supported by the Austrian Science Fund (FWF grants J2052 and J2210). I thank the Department of Mathematics of the University of Colorado at Boulder for its hospitality and especially Professor Wolfgang M. Schmidt for his support and encouragement.

\section{References}

[1] C. Baxa and J. Schoißengeier, Calculation of improper integrals using (no)-sequences, Monatsh. Math. 135 (2002), 265-277.

[2] N. G. de Bruijn and K. A. Post, A remark on uniformly distributed sequences and Riemann integrability, Indag. Math. 30 (1968), 149-150. 
[3] K. A. Driver, D. S. Lubinsky, G. Petruska and P. Sarnak, Irregular distribution of $\{n \beta\}, n=1,2,3, \ldots$, quadrature of singular integrands, and curious basic hypergeometric series, Indag. Math. (N.S.) 2 (1991), 469-481.

[4] M. Drmota and R. F. Tichy, Sequences, Discrepancies and Applications, Lecture Notes in Math. 1651, Springer, Berlin, 1997.

[5] J. H. Halton, The distribution of the sequence $\{n \xi\}(n=0,1,2, \ldots)$, Proc. Cambridge Philos. Soc. 61 (1965), 665-670.

[6] G. H. Hardy and J. E. Littlewood, Notes on the theory of series (XXIV): A curious power-series, ibid. 42 (1946), 85-90.

[7] J. Hartinger, R. F. Kainhofer and R. F. Tichy, Quasi-Monte Carlo algorithms for unbounded, weighted integration problems, J. Complexity 20 (2004), 654-668.

[8] A. Khintchine [A. Khinchin], Ein Satz über Kettenbrüche, mit arithmetischen Anwendungen, Math. Z. 18 (1923), 289-306.

[9] - Eine arithmetische Eigenschaft der summierbaren Funktionen, Recueil Math. Moscou 41 (1934), 11-13.

[10] B. Klinger, Numerical integration of singular integrands using low-discrepancy sequences, Computing 59 (1997), 223-236.

[11] C. Kraaikamp and P. Liardet, Good approximations and continued fractions, Proc. Amer. Math. Soc. 112 (1991), 303-309.

[12] L. Kuipers and H. Niederreiter, Uniform Distribution of Sequences, Wiley, New York, 1974.

[13] G. Larcher, A convergence problem connected with continued fractions, Proc. Amer. Math. Soc. 103 (1988), 718-722.

[14] J. M. Marstrand, On Khinchin's conjecture about strong uniform distribution, Proc. London Math. Soc. 21 (1970), 540-556.

[15] V. A. Oskolkov, Hardy-Littlewood problems on the uniform distribution of arithmetic progressions, Izv. Akad. Nauk SSSR Ser. Mat. 54 (1990), 159-172 (in Russian); English transl.: Math. USSR-Izv. 36 (1991), 169-182.

[16] —, The Hardy-Littlewood problem for regular and uniformly distributed sequences, Izv. Ross. Akad. Nauk Ser. Mat. 58 (1994), 153-166 (in Russian); English transl.: Russian Acad. Sci. Izv. Math. 44 (1995), 359-371.

[17] O. Perron, Die Lehre von den Kettenbrüchen, Band 1, Teubner, Stuttgart, 1977.

[18] A. M. Rockett and P. Szüsz, Continued Fractions, World Sci., Singapore, 1992.

[19] I. M. Sobol, Calculation of improper integrals using uniformly distributed sequences, Dokl. Akad. Nauk SSSR 210 (1973), 278-281 (in Russian); English transl.: Soviet Math. Dokl. 14 (1973), 734-738.

Department of Mathematics

University of Vienna

Nordbergstrasse 15

A-1090 Wien, Austria

E-mail: christoph.baxa@univie.ac.at 